\title{
NBSIR 73-330
}

\section{FREQUENCY DOMAIN MEASUREMENT OF BASEBAND INSTRUMENTATION}

N. S. Nahman and R. M. Jickling

Electromagnetics Division Institute for Basic Standards National Bureau of Standards Boulder, Colorado 80302

July 1973

Final Report

Prepared for:

Department of Defense

Calibration Coordination Group 71-52

c/o Mr. M. L. Fruechtenicht AMSMI-M, BIdg. 5435

U. S. Army Metrology \& Calibration Center

Redstone Arsenal, Alabama 35809 



\section{FREQUENCY DOMAIN MEASUREMENT OF BASEBAND INSTRUMENTATION}

N.S. Nahman and R.M. Jickling

Electromagnetics Division

Institute for Basic Standards

National Bureau of Standards

Boulder, Colorado 80302

July 1973

Final Report

Prepared for:

Department of Defense

Calibration Coordination Group 71-52

c/o Mr. M.L. Fruechtenicht AMSMI-M, BIdg. 5435

U.S. Army Metrology \& Calibration Center

Redstone Arsenal, Alabama 35809

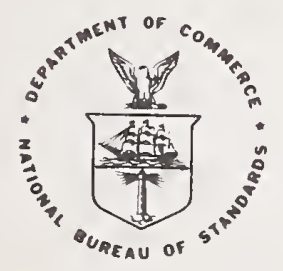

U.S. DEPARTMENT OF COMMERCE, Frederick B. Dent, Secretary 

1. INTRODUCTION

1.1 Objectives and Results 1

1.2 The Need for Frequency Domain Measurements 2

1.3 The Need for New Frequency Domain Measurement 3 Methods

1.4 Organization of the Report 4

2. NETWORK PROPERTIES OF A SAMPLING HEAD 6

2. 1 Introduction 6

2. 2 Parameters Relevant to Time Domain Analysis 6

2.3 Measurements Equivalent-Circuit Network 9

2. 4 Observability of Measurement Quantities 12

2. 5 Generality of Measurement Methods 15

2.6 A Lossless Canonical Measurement Model 16

$\begin{array}{lll}2.7 & \text { Summary } & 19\end{array}$

3. SAMPLING HEAD VOLTAGE MEASUREMENTS 20

3.1 Introduction 20

3.2 Voltage Measurements 20

3. 3 Probe Indicator Voltage Calibration 22

3.4 Experimental Results 25

4. SAMPLING HEAD INPUT IMPEDANCE MEASUREMENTS 29

4. 1 Introduction 29

4. 2 Impedance Measurement Data 29

4.3 Sampling Head Physical Structure 29

5. IMPEDANCE PERTURBATION MEASUREMENTS 34

5.1 Introduction 34

5.2 Capacitance Perturbation 34

5.3 Experimental Technique 34

5. 4 Experimental Results 35 
6. SAMPLING-LOOP IMPEDANCE MEASUREMENTS

6.1 Introduction $\quad 37$

6. 2 Sampling-Loop Impedance vs. Bias Current 40

6.3 Experimental Impedance Measurements and Results $\quad 43$

7. SUMMARY AND RECOMMENDATIONS FOR FUTURE WORK 47

7.1 Summary $\quad 47$

7.2 Recommendations for Future Work 47

$\begin{array}{ll}\text { REFERENCES } & 49\end{array}$

APPENDIX: THE ANALYSIS OF DISCRETELY LOADED 50

TRANSMISSION LINES IN TERMS OF

TRANSMITTED AND REFLECTED PULSE

TRAINS 
Figure 2.1 A typical feed-through sampling head.

Figure 2.2 General measurement network model of a feed-through sampling head.

Figure 2.3 A canonical form equivalent network for representing $\mathrm{N}_{1}$ or $\mathrm{N}_{2}$.

Figure 2.4 An equivalent circuit for the measurement

network (Fig. 2.2) in terms of cascaded admittance canonical forms.

Figure 2.5 Reduced equivalent circuit for the measurement network with sampled data network included.

Figure 2.6 Reduced equivalent circuit for the measurement network with sampled data network included assuming lossless transmission line elements.

Figure 2.7 Block diagram of the basic instrumentation for measurements on a sampling head.

Figure 3.1 Voltage probe calibration and impedance measurement system.

Figure 3.2 Slotted-section terminated in a lossless matching section $\mathrm{M}$ and a calibrated power meter $\mathrm{PM}, \mathrm{Z}_{\mathrm{p}}$ is the probe loading impedance.

Figure 3.3 Typical tuned probe equivalent circuit.

Figure 3.4 Detected probe voltage vs. transmitted power.

Figure 3.5 Typical results for an oscilloscope deflection response measurement. 
Figure 4. I Typical results for the magnitude of the input impedance, /Z/, as a function of frequency.

Figure 4.2 Typical results for the phase angle of the input impedance, $\theta$, as a function of frequency .

Figure 4.3 Sampling head physical structure.

Figure 4. 4 Loaded-transmission line equivalent network

deduced from the physical structure of the sampling head.

Figure 6.1 Balanced sampling gate across an unbalanced signal transmission line represented in a strip line geometry.

Figure 6.2 (A) Sampling loop with loop switches open.

(B) Equivalent circuit when sampling loop - switches are closed.

Figure 6.3 Equivalent circuit reduction for the measurement method of Section 2. 6 applied to the measurement of the sampling loop impedance $Z_{s}(s) / 2$.

Figure 6.4 Equivalent circuit for the sampling current loop.

Figure 6.5 Physical structure of the sampling diode package.

Figure 6.6 External bias supply connected to the diode sampling gate.

Figure 6.7 Real part of the $10 \mathrm{GHz}$ sampling loop impedance vs. sampling diode bias current.

Figure 6.8 Imaginary part of the $10 \mathrm{GHz}$ sampling loop impedance vs. sampling diode bias current.

Figure 6.9 Static I-V characteristic for a samplingdiode. 
Figure A-1 Uniform transmission line connecting an arbitrary generator and arbitrary load.

Figure A-2 The RT diagram for a uniform transmission line connecting an arbitrary generator and load.

Figure A-3 The circuit and RT diagrams for a uniform transmission line terminated at each end in its characteristic impedance $Z_{0}(s)$ and discretely loaded by the impedances $Z_{1}(s)$ and $Z_{2}(s)$. 


\section{ABSTRACT}

Microwave measurement techniques were developed for characterizing the wideband feed-through sampling heads as sociated with time domain sampling oscilloscopes and frequency domain network analyzers. Such characterization or modeling is necessary for the removal of the oscilloscope distortion from the observed waveform to yield the input waveform; also, it is useful for extrapolation in estimating oscilloscope performance at higher frequencies.

The techniques were developed through measurements on a sampling oscilloscope having a 28 picosecond transition time ( $10 \%$ to $90 \%$ ) and a $12.4 \mathrm{GHz}$ baseband bandwidth. The major results of the work are the development of voltage and impedance measurement techniques which provide the means for determining the sampling-head equivalent circuit parameters. The techniques are based upon slotted-line measurements and are not inherently li mited to any particular frequency range. Experimental results were obtained for the samplinghead input impedance over the 7-12 GHz frequency range, and for the $10 \mathrm{GHz}$ sampling loop impedance (vs. sampling-diode bias current).

Key Words: Bandwidth; diode; impedance; sampling; slotted line. 


\section{INTRODUCTION}

1.1 Objectives and Results

This is the final report on CCG project 71-52. The objective of this project was to determine how frequency domain measurements could be employed to characterize time domain baseband pulse instrumentation. The actual work was confined to the development of microwave measurement techniques for characterizing wideband feed-through sampling-heads. Such sampling heads are used for picosecond pulse oscillographic measurements and in automatic network analyzers.

The major results of the work are the development of voltage and impedance measurement techniques which provide the means for determining the parameters of equivalent-circuit networks when applied over suitable frequency ranges. The techniques are based upon slotted line measurements and are not inherently limited to any particular frequency range.

Specifically, techniques were developed for:

1. Modeling the measurement system for measurements to determine the sampling head network parameters.

2. Calibration of a slotted-line SWR detector for voltage measurements in conjunction with impedance measurements.

3. Impedance perturbation of the sampling-gate plane for equivalent network parameter determination.

4. Sampling-10op impedance measurements as a function of sampling-gate bias.

The techniques were applied in the frequency range of 7 to $12.4 \mathrm{GHz}$. 
The sampling-oscilloscope is a linear physical system operating in a sampled-data mode. By making frequency domain measurements on an operating sampling-oscilloscope and also on its component parts, experimental data may be obtained which may be used to construct a mathematical model for the oscilloscope. Such a model would describe the driving point and transfer properties of the oscilloscope in both the time and frequency domains.

The step response of a linear physical system is related to its high frequency bandwidth limitations. In principle, by Fourier Transformation methods the complete (amplitude and phase) frequency domain response could be transformed to yield the time domain (step) response. Conversely, the complete $(0 \leq t \leq \infty)$ time domain step response could be transformed to yield the frequency domain response.

In practice, the frequency or time domain responses are not completely known due to such things as (1) the inability to measure amplitude and/or phase characteristics over great enough frequency ranges, or (2) the inability to generate and observe the true step response undistorted by the generator waveform and the oscilloscope transient response.

The separation between principle and practice may be bridged by modeling the physical system. Such models essentially provide the mathematical means for extrapolating the limited measured data into time and/or frequency ranges beyond that of the experimental data. Of course, to be effective the selected model must closely represent the physical system over suitable frequency and time ranges.

Finally, because the sampling-oscilloscope is a time domain instrument, measurements in the frequency domain provide an independent means for obtaining experimental data from which a mathematical model 
could be deduced. The resultant model would have many uses. Several important ones would be as follows:

1. The computation of numerical values which describe the oscilloscope in terms of engineering and design parameters, e.g., transition time, overshoot, tilt, high frequency cut-off, bandwidth, phase-shift, etc.

2. The deconvolution of time domain waveforms, i.e., the removal of the oscilloscope distortion from the observed waveform to yield the input waveform.

3. Characterization of measurement errors.

1.3 The Need for New Frequency Domain Measurement Methods

The high frequency bandwidth limitations of a sampling-oscilloscope reside in the sampling head which consists of two major parts: (1) the electrical transmission line structure in which the sampling-gate is imbedded, and (2) the sampling-gate itself with its associated sampling current loop. Consequently, the circuit properties which must be considered include (1) the transfer of the input signal from the input terminals of the sampling-head to the sampling-gate input terminals during gate closure, and (2) the sampling processes itself, i.e., the transient response and storage properties of the sampling loop.

Since the sampling operation is a transient process of brief duration and low repetition rate, steady state measurements such as microwave impedance measurements can not observe the loading produced by the sampling-gate. This point can be simply illustrated by making a $10 \mathrm{GHz}$ measurement of the sampling-head input impedance with and without triggering the oscilloscope.

Because the sampling-gate input terminals are imbedded within a nonuniform transmission line network, signal transfer from a $50 \mathrm{ohm}$ 
system to the sampling-head input terminals is different from that to the sampling-gate input terminals. Furthermore, the transfer functions from the sampling-head input and the sampling-gate input, respectively, to the oscilloscope display a re also different.

As a consequence of the above listed properties, new measurement methods are needed; specifically, techniques for determining the following quantities are required:

1. voltage and impedance at the sampling-head input reference plane.

2. voltage and impedance at the sampling-gate plane.

3. impedance of the sampling-loop as a function of the samplinggate biasing current.

1.4 Organization of the Report

This report is divided into six chapters, the first chapter being the present introduction. Chapters 2 through 6 report the technical work while Chapter 7 presents a summary and recommendations for future work. The technical contents of Chapters 2 through 5 a re briefly described below.

Chapter 2 presents a general measurement network model for measurements on the sampling-head. The measurement data presented in chapters 3-5 illustrate the application of the measurement model.

Chapter 3 presents the basic voltage and impedance measurement system which consists of a slotted-line section and a calibrated voltage probe. The theory and application of the measurement techniques are discussed along with experimental examples of measurements and probe calibration. 
Chapter 4 presents the results of sampling-head input impedance measurements. The results are correlated with the physical structure of the sampling-head.

Chapter 5 presents the technique for immittance perturbation of the sampling-gate reference plane and the corresponding effect at the sampling-head input reference plane.

Chapter 6 presents the technique for impedance measurements on the sampling-loop as a function of the sampling-gate bias. Experimental data are presented.

Chapter 7 is a summary and includes recommendations for future work.

An appendix is included to explain the passage of transmitted and reflected pulse trains in a discretely loaded transmission line. 


\section{NETW ORK PROPERTIES OF A SAMPLING HEAD}

\section{1 Introduction}

The high frequency bandwidth limitations of a sampling oscilloscope reside in the sampling head which consists of two major parts: (1) the electrical transmission line structure in which the (sampling) gate is imbedded, and (2) the gate itself with its associated sampling current loop. Consequently, the circuit properties being considered here include (1) the transfer of the (sampling) head input signal to the gate input terminals during the gate closure, and (2) the sampling process itself. The former necessarily includes the gate input terminal admittance during gate closure.

\subsection{Parameters Relevant to Time Domain Analysis}

A typical head circuit represented as a transmission line network is shown in Fig. 2. 1. The circuit elements shown in Fig. 2. 1 are defined as follows:

$$
\begin{aligned}
\mathrm{Y}_{\mathrm{c}} \equiv & \text { Input or output connector-pair discontinuity } \\
& \text { admittance } \\
\mathrm{Y}_{\mathrm{g}} \equiv & \text { Gate input admittance (varies with gate state) } \\
\mathrm{Y}_{\mathrm{b}} \equiv & \text { Low frequency (blow-by) compensator probe } \\
& \text { admittance } \\
\mathrm{Y}_{0} \equiv & \text { Characteristic admittance of the uniform input or } \\
& \text { output transmission lines. }
\end{aligned}
$$

Typically, the transmission line lengths $\ell_{2}, l_{3}$, and $l_{4}$ are different and are each of the order of $5 \mathrm{~cm}$. 


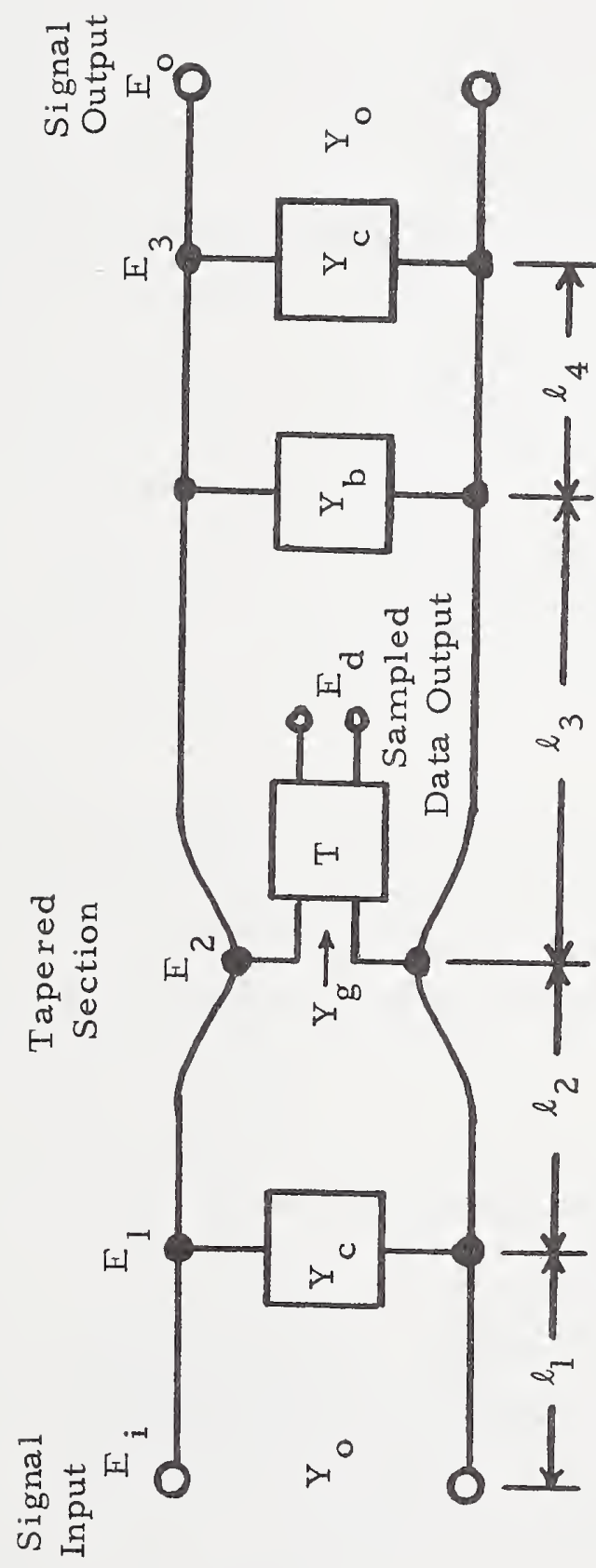

年 
The frequency domain parameters which are paramount for characterizing the time domain (pulse) response of a sampling head a re different from those terminal network parameters used in characterizing microwave networks. For time domain considerations, parameters internal to the network (sampling head) terminals are paramount and convenient in application. That such is the case arises from the fundamental fact that a narrow pulse or impulse traveling through a discretely-loaded uniform transmission line encounters each load (discontinuity) separately, successively in increasing time. With each encounter, the transmitted pulse waveform is altered and a reflected waveform is generated. Overall, the output from the discretely loaded line of length $\ell$ will consist of a train of pulses, the first of which having the Laplace Transform (Appendix I)

$$
\left[E_{2}(s)\right]=E_{1}(s) \prod_{n=1}^{m} \frac{2 Y_{0}(s) e^{-l_{n} \gamma(s)}}{Y_{n}(s)+2 Y_{0}(s)}
$$

Initial Pulse

where $\mathrm{Y}_{n}(\mathrm{~s})$ is the $\mathrm{n}$-th discrete load and $\mathrm{Y}_{0}(\mathrm{~s})$ the uniform line characteristic admittance. The product of the $\mathrm{m}$ factors in (2-1) corresponds to the cascaded effects of $\mathrm{m}$ discontinuities (discrete loads). The expressions corresponding to pulses following the first output pulse will contain reflection factors.

Equation (2-1) may be applied to the analysis of a sampling head. In Fig. 2. 1, assuming that the tapered section maintains the characteristic admittance $\mathrm{Y}_{0}$, then by $(2-1)$ the initial pulse appearing at the sampled output has the Laplace Transform

$$
E_{d}(s)=4 E_{i}(s) \frac{G_{o} e^{-l_{1} \gamma(s)}}{Y_{c}(s)+2 G_{0}} \frac{G_{o} e^{-l_{2} \gamma(s)}}{Y_{g}(s)+2 G_{0}} T(s)
$$


where the impedances have been written as admittances. $Y_{0}$ has been assumed to be a real conductance $G_{0}$ (i. e., lossless line), and $T(s)$ is the sampler transfer function. The time domain response corresponding to $(2-2)$ is valid for the time before reflections from $Y_{c}$ and $Y_{b}$ return to the $E_{2}$ plane. Since $\ell_{2}$ and $\ell_{3}$ are approximately $5 \mathrm{~cm}$ (air dielectric), the first reflections will arrive in $334 \mathrm{ps.} \mathrm{Consequently,} \mathrm{the}$ inverse Laplace Transform of $(2-2)$ is valid for the time interval $0 \leq \mathrm{t} \leq 334 \mathrm{ps}$.

The significance of $(2-2)$ is now apparent. If $\mathrm{Y}_{c}(s), \mathrm{Y}_{\mathrm{g}}(\mathrm{s})$ and $T(s)$ are known in the $s$ domain, then $e_{d}(t)$ for $0<t<334$ ps may be compared to (2-2) through $\mathcal{L}_{\mathrm{d}}(t)=\mathrm{E}_{\mathrm{d}}(\mathrm{s})$. Furthermore, only $Y_{c}$ (at the input) and $Y_{g}$ in conjunction with $G_{0}$ govern the transfer of the pulse from the uniform input line to the gate input plane, during the time interval $0<\mathrm{t}<334 \mathrm{ps}$.

These results are in marked contrast to the steady state situation in which the cumulative effects of all reflections combine to produce steady state transfer functions. Clearly, it is evident that microwave measurements which directly determine $Y_{c}(s), Y_{g}(s)$ and $T(s)$ are needed.

\section{3 Measurements Equivalent-Circuit Network}

The circuit properties being considered here are those primarily related to the transfer of a signal from a node on a uniform input trans mission line to the sampling-gate input node. This necessarily includes the effect of the sampling-gate input admittance during the gate closure.

For measurement purposes it is convenient to define three nodes or reference planes of a general network, Fig. 2.2: 


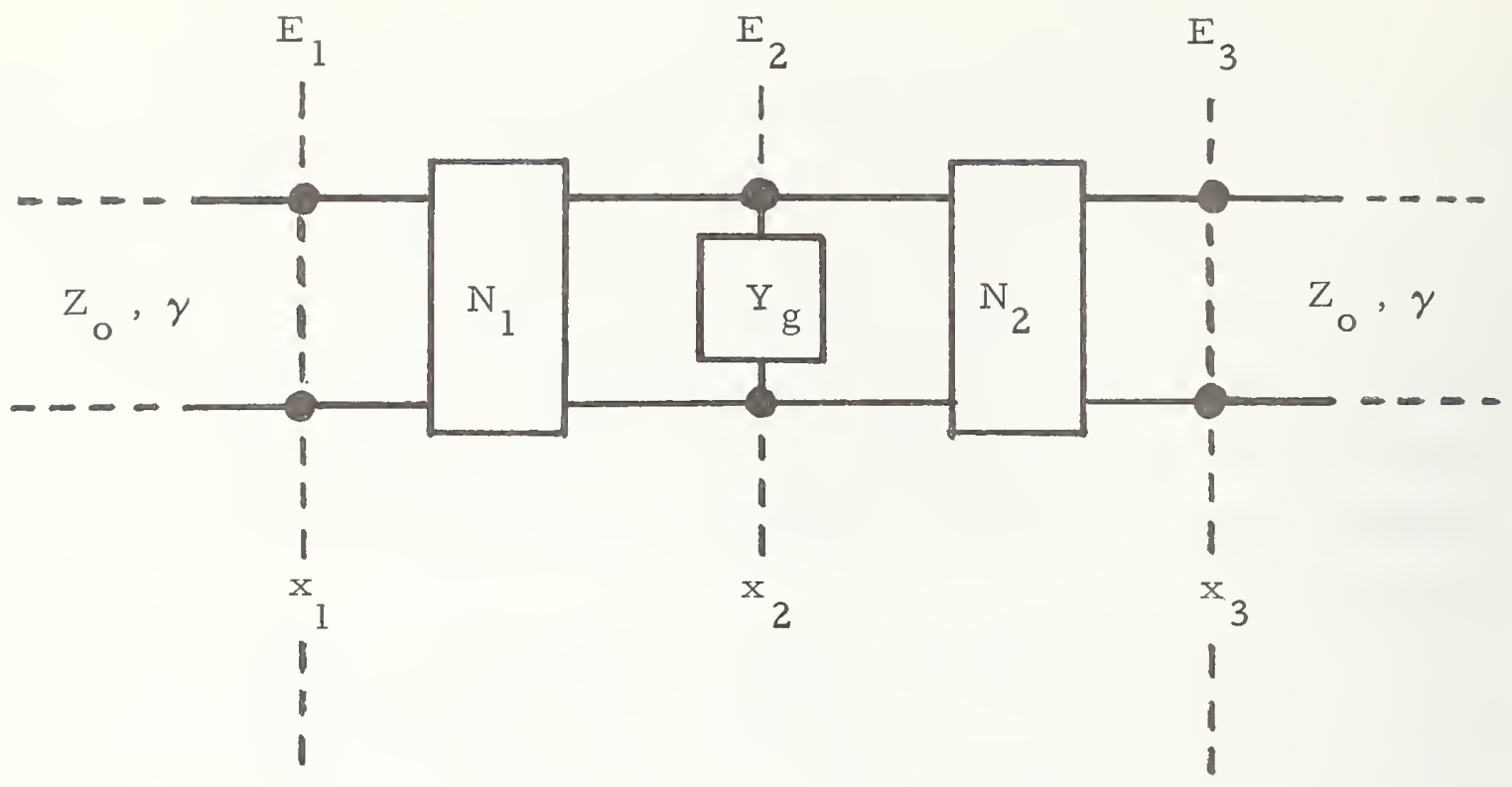

Uniform Trans mission Line
Lumped Equivalent

Networks
Uniform Trans mission Line

Figure 2.2 General measurement network model of a feed-through sampling head.

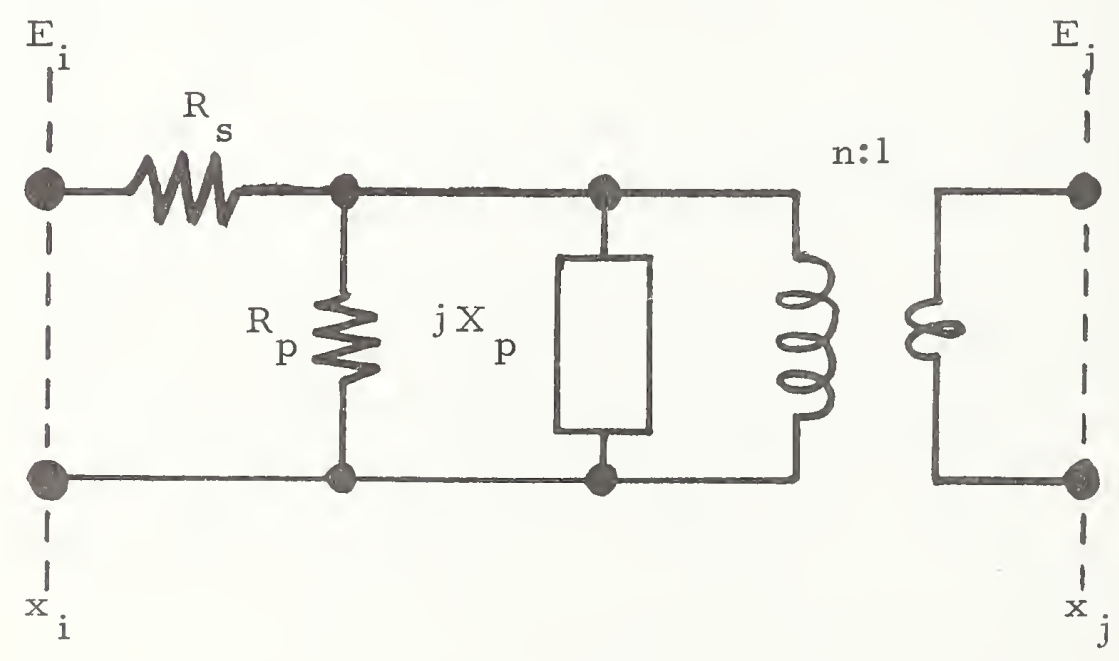

Figure 2.3 A canonical form equivalent network for representing $\mathrm{N}_{1}$ or $\mathrm{N}_{2}$. 
1. $\mathrm{x}_{1}$, a plane (or node $\mathrm{E}_{1}$ ) in a uniform transmission line before the sampling head input terminals.

2. $x_{2}$, a plane (or node $E_{2}$ ) internal to the sampling-head at the sampling-gate input node.

3. $x_{3}$ a plane (or node $E_{3}$ ) in a uniform transmission line following the sampling head (feed-through) output terminals.

The three defined planes are connected together by the lumped equivalent networks $\mathrm{N}_{1}$ and $\mathrm{N}_{2}$, and the equivalent admittance $\mathrm{Y}_{\mathrm{g}}$. That is, in Fig. 2.2 to left and right of the planes $x_{1}$ and $x_{3}$ respectively, uniform transmission lines are defined, while between the planes $x_{1}$ and $x_{3}$ lumped equivalent networks are defined:

1. $\mathrm{N}_{1}$ is an equivalent lumped network representing the network properties between the reference planes $x_{1}$ and $x_{2}$. $\mathrm{N}_{1}$ includes any waveguide to transmission line transitions, sampling-head input connectors, and networks internal to the sampling-head leading to the reference plane $x_{2}$ from ${ }^{x}{ }_{1}$

2. $Y_{g}$ is the sampling-gate input admittance located at the plane $x_{2}$.

3. $\mathrm{N}_{2}$ is an equivalent lumped network representing the network properties between the reference planes $x_{3}$ and $x_{2}$. $\mathrm{N}_{2}$ includes any waveguide to transmission line transitions, sampling-head input connectors, and networks internal to the sampling-head leading to the reference plane $x_{2}$ from $\mathrm{x}_{3}$. 
The equivalent circuit networks $\mathrm{N}_{1}$ and $\mathrm{N}_{3}$ can be of the canonical form shown in Fig. 2.3 [1]. Consequently, the general measurement network of Fig. 2.2 in terms of the canonical form in Fig. 2. 3 would contain a cascade of two such networks. In terms of admittance elements Fig. 2. 4 results and may be reduced to that of Fig. 2. 5 in which

$$
\begin{aligned}
& \mathrm{Y}_{2}=\mathrm{G}_{2}+j B_{2} \\
& \mathrm{G}_{2}=\mathrm{n}_{1}^{2} G_{\mathrm{pl}}+\mathrm{n}_{2}^{2} \mathrm{G}_{\mathrm{p} 2}+\mathrm{G}_{\mathrm{g}} \\
& \mathrm{B}_{2}=\mathrm{n}_{1}^{2} \mathrm{~B}_{\mathrm{pl}}+\mathrm{n}_{2}^{2} \mathrm{~B}_{\mathrm{p} 2}+\mathrm{B}_{\mathrm{g}}
\end{aligned}
$$

Also, beyond the considerations discussed in relation to Fig. 2. 4, the sampled-data circuit has been included in Fig. 2.5. $\mathrm{Y}_{2}$ contains the driving point admittance of the sampled data network while $T(s)$ is the transfer function from the real time voltage $E_{2}$ to the sampled-data voltage $\mathrm{E}_{\mathrm{d}}$.

\subsection{Observability of Measurement Quantities}

Referring to Fig. 2.5 at a given frequency there are three fully observable quantities; they are the driving point impedance and driving point voltage at each of the reference planes $x_{1}$ and $x_{3}$, and the sampled-data display voltage $\mathrm{E}_{\mathrm{d}}$.

$\mathrm{E}_{2}$ is not an observable quantity; generally, it may be deduced from a measurement of a driving point voltage and a knowledge of those equivalent circuit parameters relevant to the conditions of the voltage measurement. However, it can be simply deduced when $E_{d}$ is zero because $E_{\mathrm{d}}$ is directly proportional to $\mathrm{E}_{2}$. 

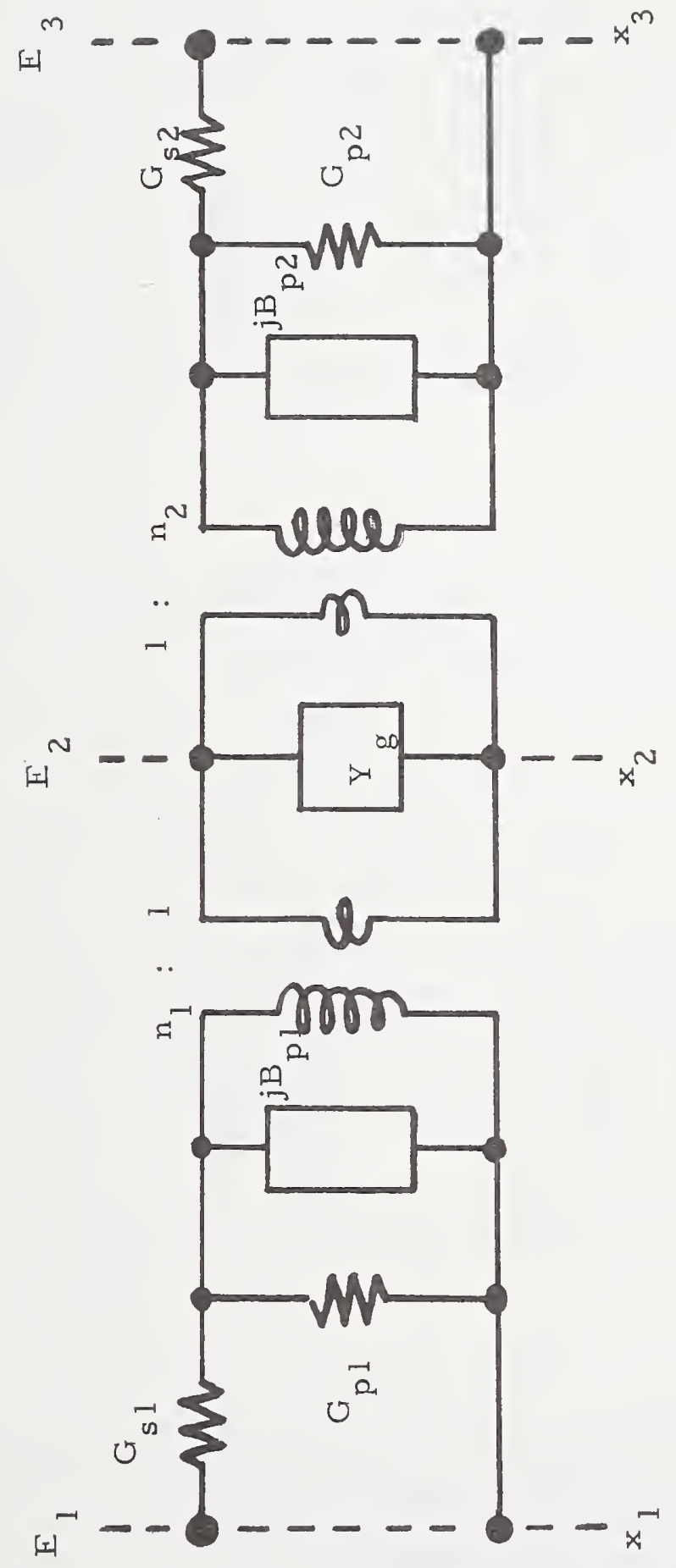

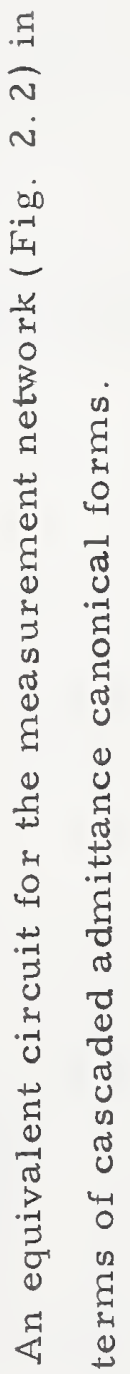

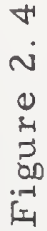




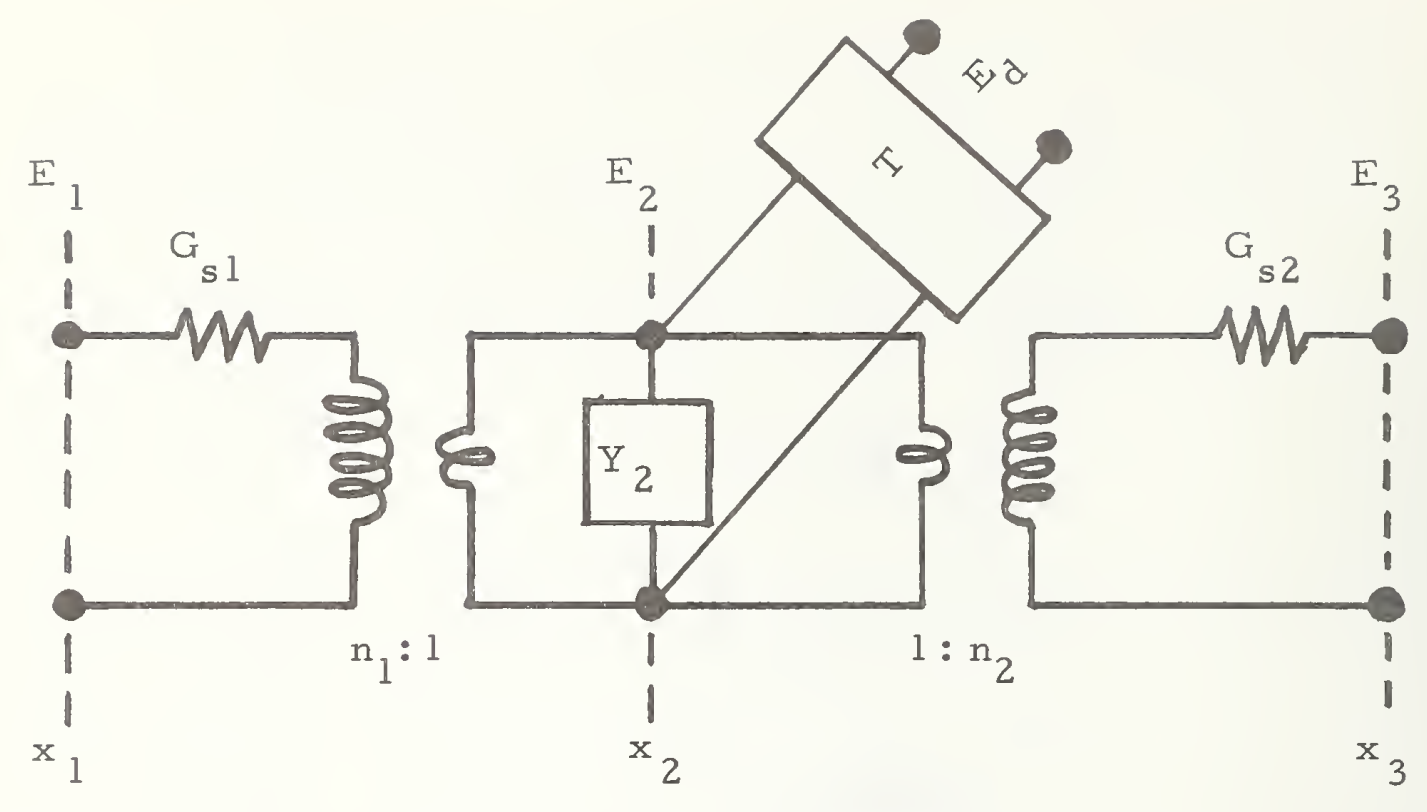

Figure 2.5 Reduced equivalent circuit for the measurement network with sampled data network included.

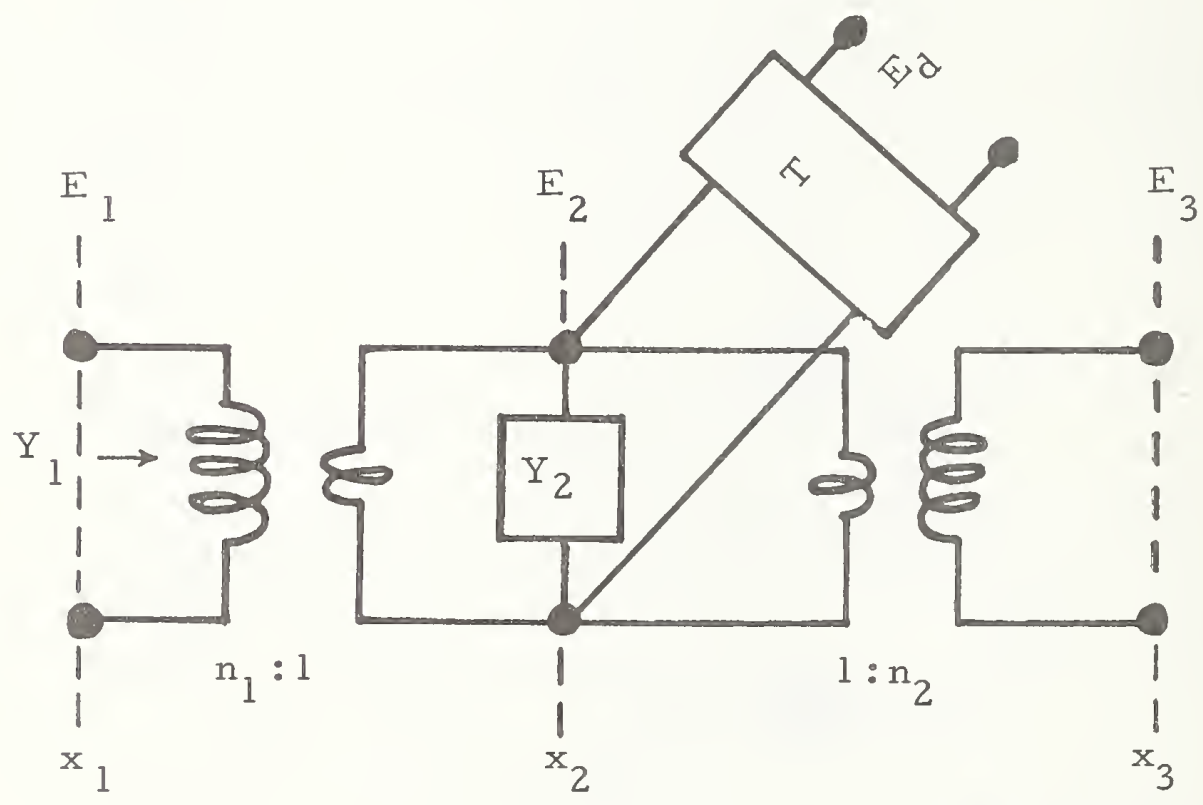

Figure 2.6 Reduced equivalent circuit for the measurement network with sampled data network included assuming lossless transmission line elements. 


$$
E_{d}=E_{2} T
$$

If $\mathrm{E}_{2}$ has been deduced from experimental data, then by virtue of (2-6), the magnitude of the sampling-gate voltage transfer function $|T|$ can be deduced as $E_{d}$ is observable.

Also, the admittance $\mathrm{Y}_{2}$ is not an observable quantity; it must be deduced from driving point admittance measurements.

And finally, the equivalent circuit parameters are generally not observable quantities but must be deduced from a set of driving point immittance measurements.

\subsection{Generality of Measurement Methods}

In light of the observability conditions delineated in Section 2. 4, the equivalent circuit parameters of Fig. 2.5 can be deduced by application of the general immittance measurement methods for canonical networks [2] in combination with voltage measurements.

Because the sampling-gate is imbedded in a transmission line structure which is tapered down to small dimensions, the sampling head losses are not negligible at the upper frequencies of operation, $18 \mathrm{GHz}$. Consequently, at the upper frequencies the general measurement methods [2] must be used which include losses in the networks, $\mathrm{N}_{1}$ and $\mathrm{N}_{2}$, Fig. 2.2. However, at $10 \mathrm{GHz}$ the losses are relatively minor and, simpler (lossless) methods may be employed.

In the experimental work reported in this report (Chapters 5 and 6), only the lossless methods were used as the frequency was restricted to about $10 \mathrm{GHz}$. This was done because the emphasis of the work was to develop the technique and demonstrate it in a simple manner. In the next section measurement methods are discussed which 
are based upon lossless canonical networks.

2.6 A Lossless Canonical Measurement Mode1

When $\mathrm{N}_{1}$ and $\mathrm{N}_{2}$ in Fig. 2.2 are lossless, then the canonical network in Fig. 2.3 reduces to a lossless one, i. e., $R_{S}$ vanishes and $R_{p}$ becomes infinite. Accordingly, the reduced equivalent circuit becomes that of Fig. 2.6 with the components of $\mathrm{Y}_{2}$ becoming

$$
\begin{aligned}
& \mathrm{G}_{2}=\mathrm{G}_{\mathrm{g}} \\
& \mathrm{B}_{2}=\mathrm{n}_{1}^{2} \mathrm{~B}_{\mathrm{p} 1}+\mathrm{n}_{2}^{2} \mathrm{~B}_{\mathrm{p} 2}+\mathrm{B}_{\mathrm{g}}
\end{aligned}
$$

Note that here the transmission line elements (measurement system components) have been assumed to be lossless. However, the samplinggate elements are lossy; consequently, $\mathrm{Y}_{2}$ and $\mathrm{T}$ remain complex.

When the lossless transmission line conditions are satisfied, the immitance seen at reference plane $x_{1}$ is simply related to that at plane $x_{2}$ through the turns ratio $n_{1}$. Specifically, in Fig. 2.6 the admittance seen at the reference plane $x_{1}$ would be given by

$$
\mathrm{Y}_{1}=\mathrm{Y}_{2} / \mathrm{n}_{1}^{2}
$$

where $\mathrm{Y}_{2}$ is the total load admittance at reference plane $\mathrm{x}_{2}$, and $\mathrm{n}_{1}$ is the transformer turns-ratio. Also, $E_{1}$ would be related to $E_{2}$ by

$$
E_{1}=E_{2} / n_{1}
$$


To establish the proper reference planes [3] so that the equivalent circuit in Fig. 2.6 is valid, a slotted-transmission line section is connected to the input terminals of the sampling head while the feedthrough output terminal is terminated in $50 \mathrm{ohms}$. With the slottedsection excited from an isolated CW source, the oscilloscope is placed in normal operation so as to display the CW waveform. Caution, the peak to peak value of the $\mathrm{CW}$ waveform should not exceed the maximum ratings specified for the sampling head (usually about one volt $\mathrm{p}-\mathrm{p}$ ). Then, the $50 \mathrm{ohm}$ output termination is replaced by a sliding short. Fig. 2. 7 shows the block diagram of the basic instrumentation system for measurements on a sampling head. $x_{1}, x_{2}$, and $x_{3}$ denote positions corresponding to those in Fig. 2.6.

The sliding-short is adjusted so that the oscilloscope display $E_{d}$ goes to zero indicating a voltage zero $\left(\mathrm{E}_{2}=0\right)$ at the sampling-gate reference plane $x_{2}$; the sliding short position defines $x_{3}$. The slotted-section probe position is moved to a voltage zero, the position of which defines $x_{1}$. With $x_{1}$ so determined the admittance and voltage seen at $x_{1}$ will be related to those at $x_{2}$ through (2.9) and (2.10), respectively. Consequently, if the sliding short were to be shifted by a quarter-wavelength, then the admittance seen at $x_{1}$ would be

$$
\frac{\left.\mathrm{Y}_{1}\right]}{\mathrm{x}_{3}+\lambda / 4}=\mathrm{Y}_{2} / \mathrm{n}_{1}^{2}
$$

because an open circuit would be established across $Y_{2}$. Also, the short could be shifted to a position so that $Y_{1}$ would be real which would mean that the residual susceptances at $x_{2}$ were canceled out. 


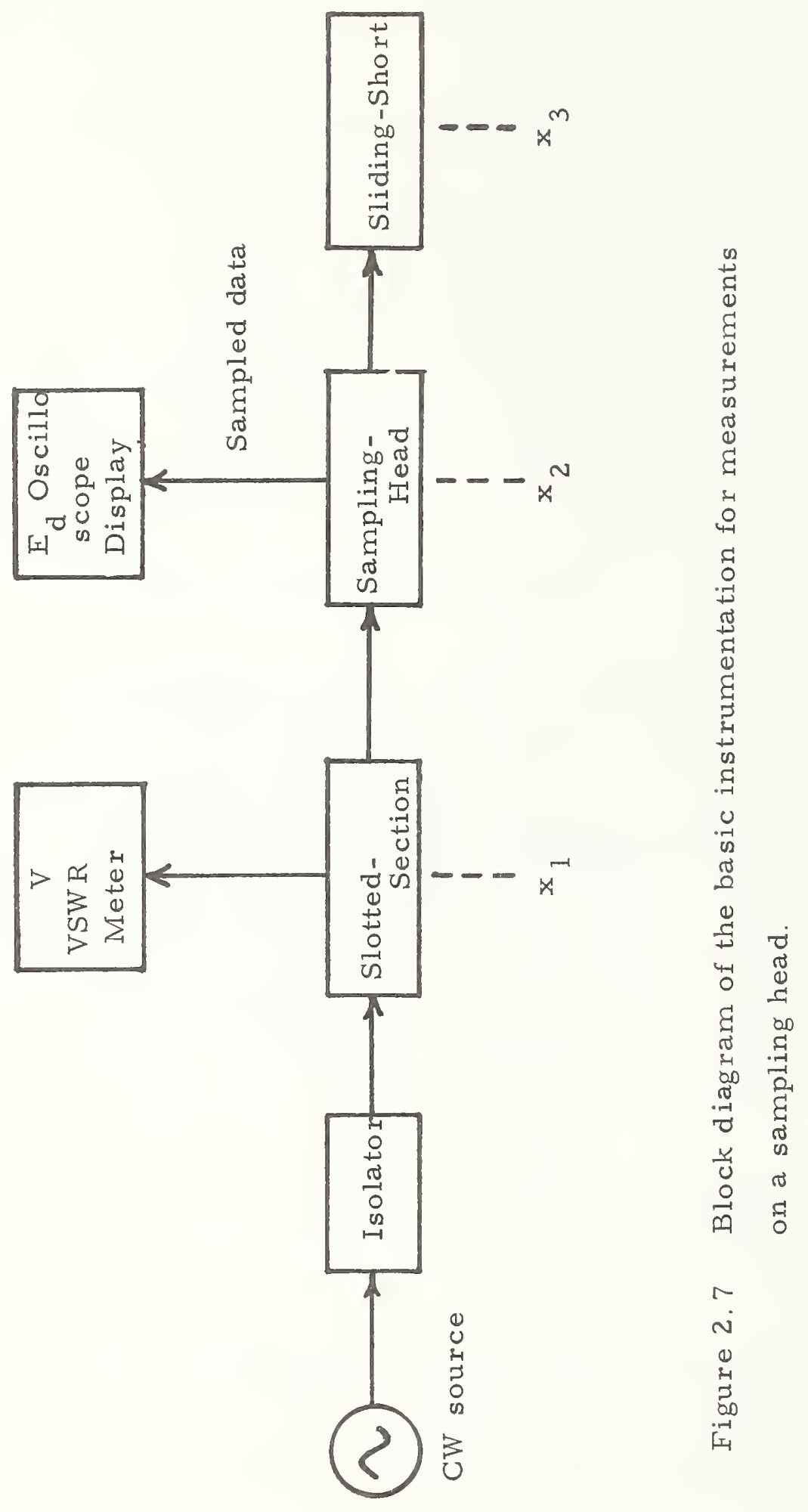


Using an immittance perturbation method, the turns ratio $\mathrm{n}_{1}$ may be determined. For example, referring to (2-9), if a known admittance perturbation $\Delta$ is introduced at $x_{2}$, then the admittance seen at $x_{1}$ is

$$
Y_{1}+\delta=\frac{1}{n_{1}^{2}}\left(Y_{2}+\Delta\right)
$$

where $\delta$ is the corresponding change in the admittance $\mathrm{Y}_{1}$. Consequently, $\mathrm{n}_{1}$ can be determined by

$$
\mathrm{n}_{1}=(\Delta / \delta)^{1 / 2}
$$

because $\Delta$ is a known admittance perturbation while $\delta$ is an observed admittance change.

\section{2. $7 \quad$ Summary}

A general measurement network model for measurements on a feed-through sampling-head has been presented. A reduced equivalent circuit for the measurement network was developed and discussed in some detail for the lossless case.

In the next chapter, the basic voltage and impedance measurement system will be discussed. Experimental results will be presented for frequencies in which the lossless measurement system assumptions are valid ( 7 to $12.4 \mathrm{GHz}$.). 


\section{SAMPLING-HEAD VOLTAGE MEASUREMENTS}

\subsection{Introduction}

The voltage and impedance measurement system is a slottedline system in which the detected probe voltage is calibrated in respect to transmission line voltage at the probe position. The probe output voltage is calibrated by establishing a unity standing-wave ratio in the slotted section and computing the slotted-section line voltage from a measurement of the transmitted power. A typical measurement system is shown in Fig. 3.1.

\subsection{Voltage Measurements}

At a given probe position in the slotted section $l$ meters from the load, $Z_{L}$, at the reference plane, the voltage on the slotted section is given by

$$
\begin{aligned}
& E(l)=E^{+}+E^{-} \\
& E(l)=\frac{E_{L}}{1+\rho} e^{\gamma^{l}}+\frac{E_{L} \rho}{1+\rho} e^{-\gamma l}
\end{aligned}
$$

where $\rho$ is the voltage reflection coefficient

$$
\rho=\frac{Z_{L}-Z_{O}}{Z_{L}+Z_{O}}
$$

and $E_{L}$ is the voltage at the load reference plane. $\gamma$ is the propagation constant of the slotted section and $z_{0}$ is its characteristic impedance. In (3.2) the first term is the incident voltage $\mathrm{E}^{+}$, while the second term is the reflected voltage $\mathrm{E}^{-}$. 


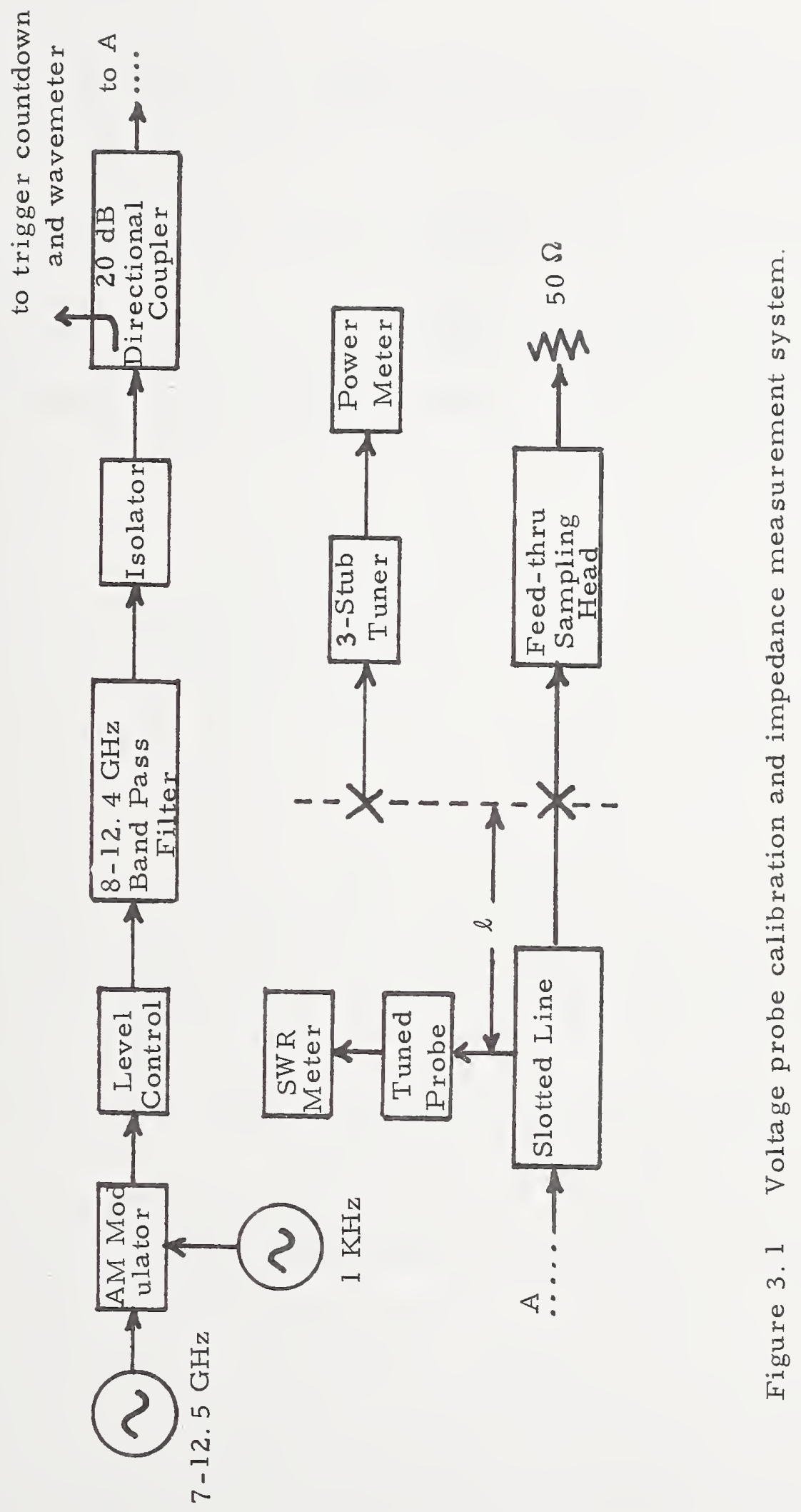


For a lossless line $\gamma=\mathrm{jB}$; if $\beta l=\pi$ radians, $l$ is equal to a half-wavelength $\lambda / 2$ at the operating frequency; and (3.2) reduces to

$$
E(\lambda / 2)=-E_{L}
$$

Consequently, the voltage at $\lambda / 2$ meters from the load reference plane is equal to the negative of that across the load $Z_{L}$. In general, the detected probe voltage $E_{p}$ is proportional to the magnitude of the line voltage $E(\lambda / 2)$,

$$
E_{p}=K|E(\lambda / 2)|
$$

where $K$ is a scalar quantity which may depend upon the magnitude of $|E(\lambda / 2)|$. Therefore, if $K$ is known, the slotted line measurements provide a means for computing the incident voltage magnitude $\left|E^{+}\right|$, i. e.,

$$
\left|E^{+}\right|=\frac{E_{p}}{K|1+\rho|}
$$

$\rho$ is computed from (3.3) using the value of $Z_{L}$ observed by the slottedsection using the null-shift method for impedance measurement [4].

3.3 Probe Indicator Voltage Calibration

$\mathrm{K}$ is determined by the probe calibration procedure. The probe indicator is calibrated by terminating the slotted-section in its characteristic impedance $Z_{0}$ using a lossless matching-section and a calibrated power meter as a load. The equivalent circuit for the calibration procedure is shown in Fig. 3.2. $M$ is adjusted so that the VSWR on the slotted section is unity. For negligible losses in the slotted-section and matching section, the power transmitted along the 
Slotted Section

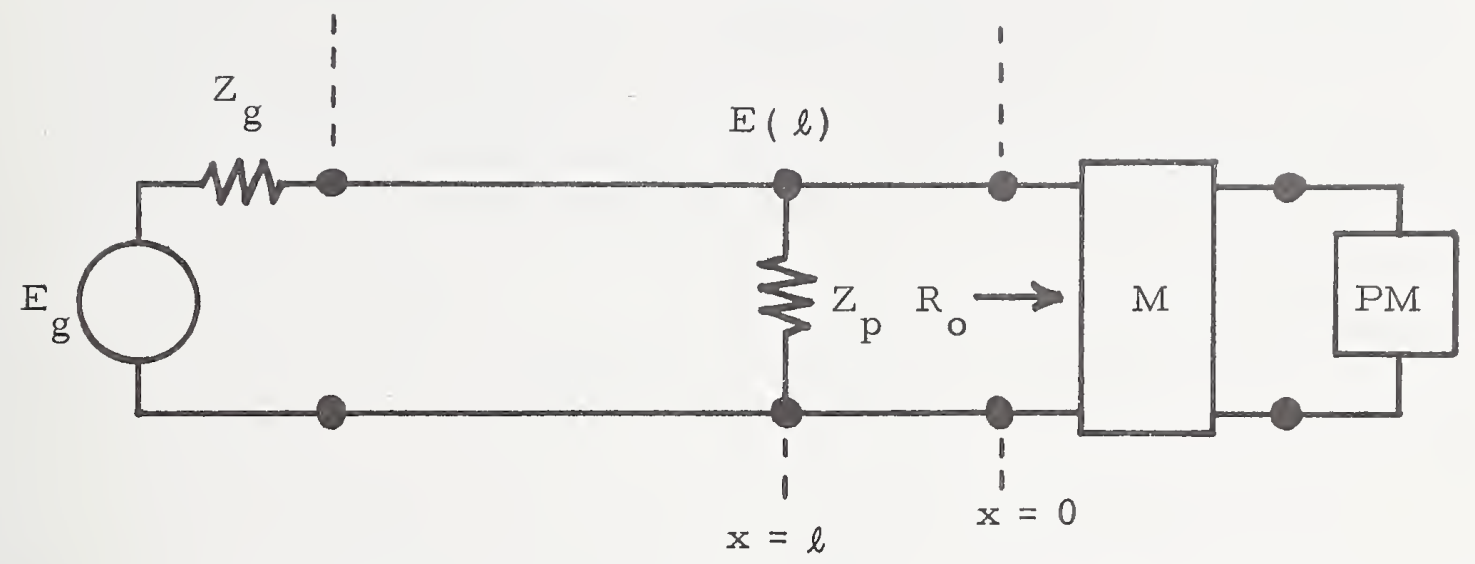

Figure 3.2 Slotted-section terminated in a lossless matching section $M$ and a calibrated power meter PM. $Z_{p}$ is the probe loading impedance.

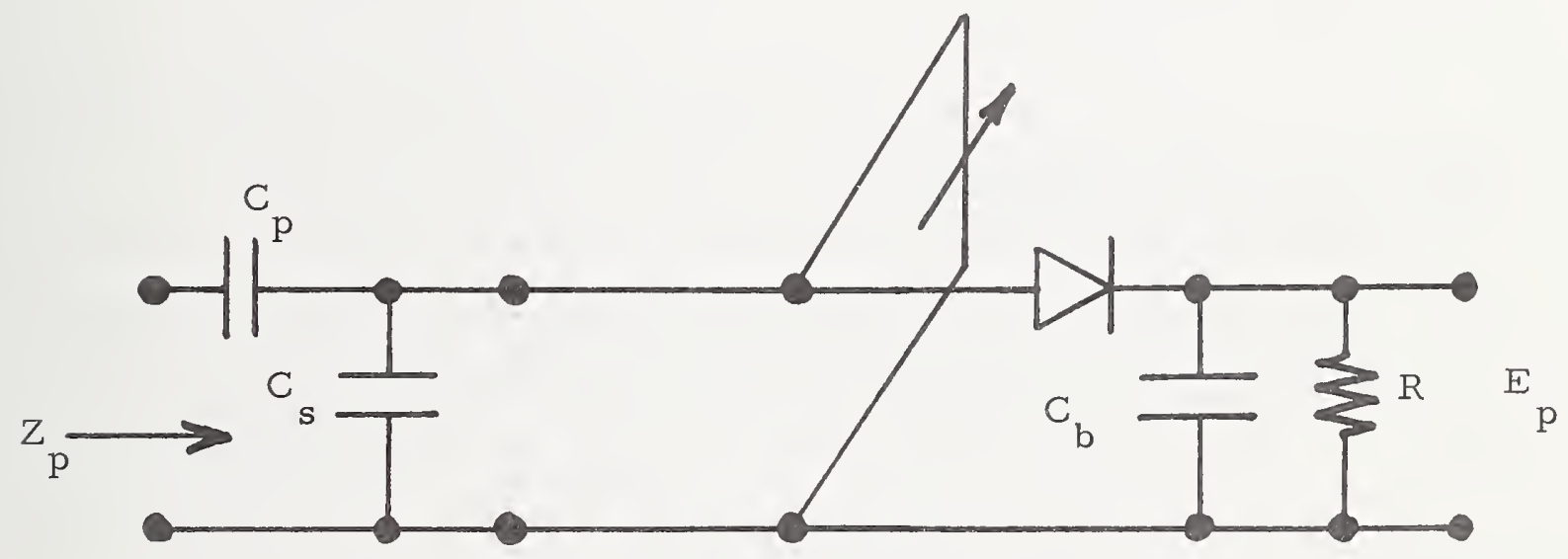

Figure 3.3 Typical tuned probe equivalent circuit. 
slotted-section, through the matching-section, and absorbed in the power meter load will each be the same. Accordingly, for power P transmitted, the transmission line voltage magnitude $|E(x)|$ everywhere in the interval $0 \leq \mathrm{x} \leq l$ will be independent of position $\mathrm{x}$ and given by

$$
|E(l)|=\sqrt{P R_{0}}
$$

and

$$
|E(l)|^{2}=P R_{0}
$$

Now in (3.5), $\mathrm{K}$ in general may depend upon the magnitude of the line voltage. Commonly, $K$ exhibits a linear dependence on $E$ for a square-law detector, i.e., for

$$
K=K^{\prime} E(\ell)
$$

the detected probe voltage is

$$
E_{p}=K^{\prime}|E(\ell)|^{2}
$$

or

$$
E_{p}=K^{\prime} R_{0} P
$$

which is proportional to power P.

For the probe calibration to be valid, care must be taken to insure that the probe coupling, tuning, etc. are constant, i.e., the same values are present during a measurement run as were present during the calibration.

Structural stability and uniformity will insure that the equivalent probe coupling parameters are constant; however, small changes in 
frequency will alter the probe tuning which in turn will change the value of $K$ in (3.5) from that of the calibration. A typical probe equivalent circuit is shown in Fig. 3.3 in which

$$
\begin{aligned}
C_{p} \equiv & \text { effective coupling capacitance to electric field } \\
& \text { in slotted line } \\
C_{s} \equiv & \text { capacitance of probe to transmission line slotted- } \\
& \text { surface } \\
C_{b} \equiv & \text { diode by-pass capacitor } \\
R & \text { detector load resistor. }
\end{aligned}
$$

The stub is adjusted to maximize the dc voltage $E_{p}$. Frequency shifts cause corresponding changes of $E_{p}$; the magnitude of such changes depend upon the effective $Q$ of the probe circuit.

\subsection{Experimental Results}

A typical probe calibration curve is shown in Fig. 3.4; here, the detector had square-law characteristics as is seen by the linear relation between transmitted power and detected probe voltage. The transmission line voltage is related to the detected probe voltage through (3.9),

where

$$
E(\ell)=\sqrt{K^{\prime} E_{p}}
$$

$$
\begin{aligned}
K^{\prime} & =\frac{E_{p}}{R_{0} P} \\
& =\frac{200 \times 10^{-6}}{50 \times 3.2 \times 10^{-3}}=1.25 \times 10^{-3}
\end{aligned}
$$




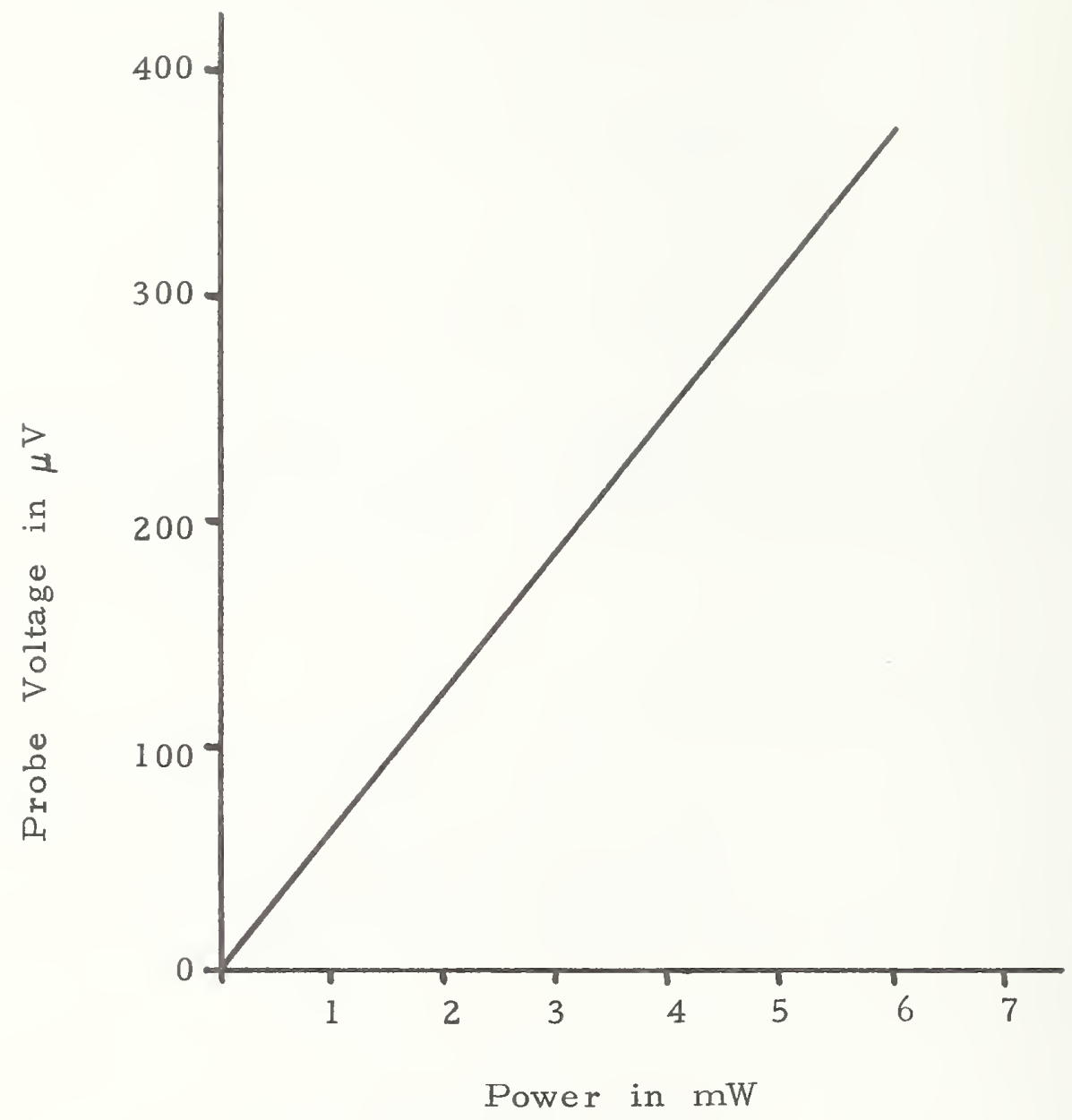

Figure 3.4 Detected probe voltage vs. transmitted power. 
Figure 3.5 shows the typical results for an oscilloscope deflection response measurement vs. the incident voltage $\mathrm{E}^{+}$at the samplinghead input port (curve A). Note that oscilloscope response saturates and only remains linear up to about $E_{d}=1.0$ volts peak to peak which corresponds to 0.35 volts RMS (this particular sampling-head has a maximum rating of $1.0 \mathrm{~V} \mathrm{p}-\mathrm{p}$; consequently, the measurements . reported here exceed the safe range). 


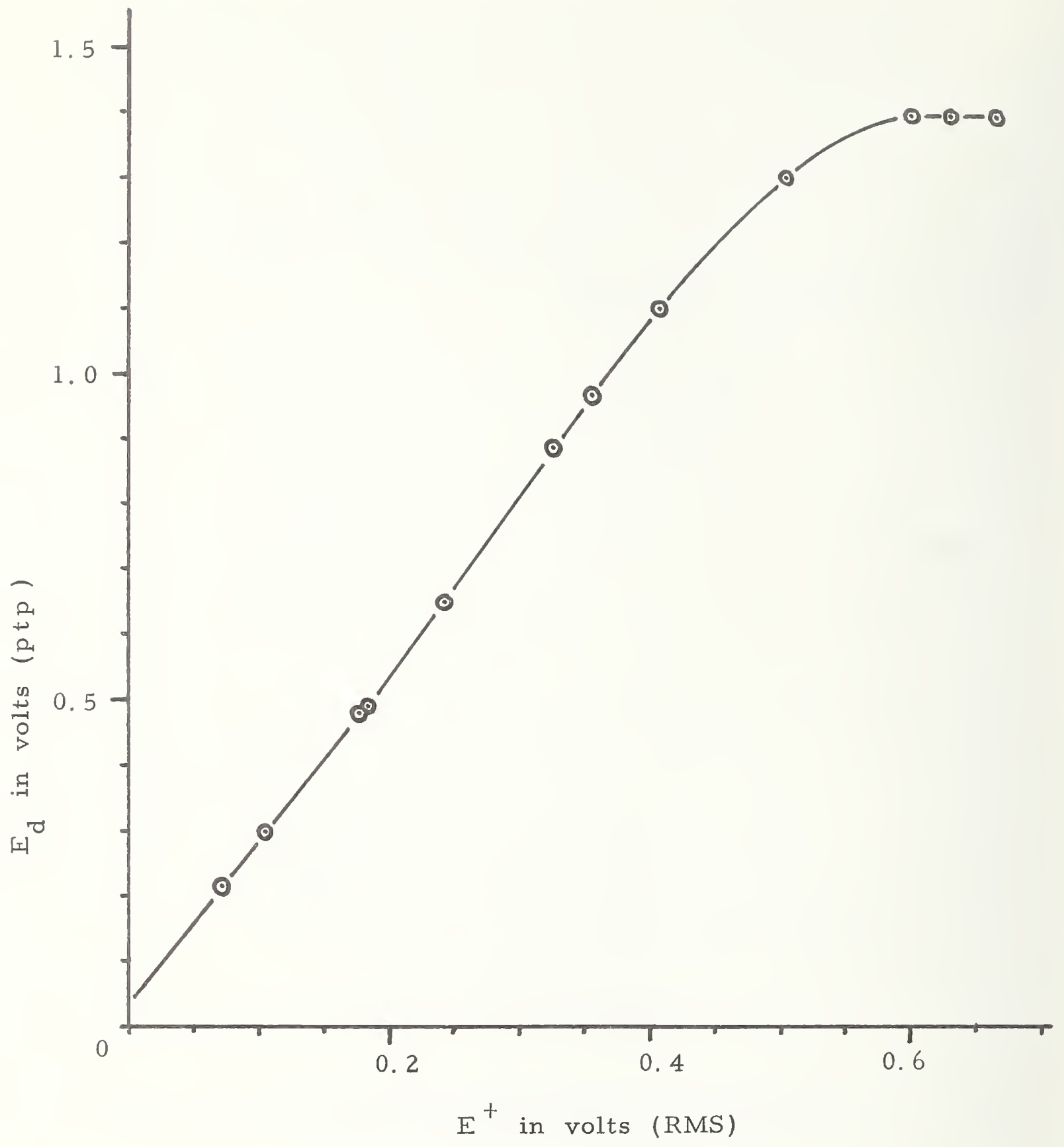

Figure 3.5 Typical results for an oscilloscope deflection response measurement. 


\section{SAMPLING HEAD INPUT IMPEDANCE MEASUREMENTS}

4. $1 \quad$ Introduction

Using the sampling head input port as a reference plane, steadystate impedance measurements can be easily made. In this chapter, typical results of slotted-line impedance measurements are presented along with a brief discussion of the frequency dependence due to the sampling head physical structure.

4.2 Impedance Measurement Data

Input port impedance measurements were made on a sampling head over the frequency range of 7.0 to $12.4 \mathrm{GHz}$. The output port was terminated in a nominal $50 \mathrm{ohm}$ load as provided by a $50 \mathrm{ohm}$ termination specified by the sampling-head manufacturer. The measurements were made with the system shown in Fig. 3. 1.

Typical magnitude and phase data for the input impedance are shown in Figs. 4. 1 and 4.2, respectively. The magnitude and phase data indicate that discontinuities are present in the $50 \mathrm{ohm}$ transmission line structure which comprises the sampling head. Note that the oscillation of the data contains a fine-structure ripple. The major oscillation has a period of about $2.5 \mathrm{GHz}$ measured between the zero crossings of the phase angle curves. The ripple in the roughly periodic data suggests that the physical structure of sampling-head is nearly periodic.

\section{3 Sampling-Head Physical Structure}

As a physical structure, the sampling head consists of a cascade of three transmission line sections and consequently four connection planes. At each connection plane, discontinuities are present. Starting 


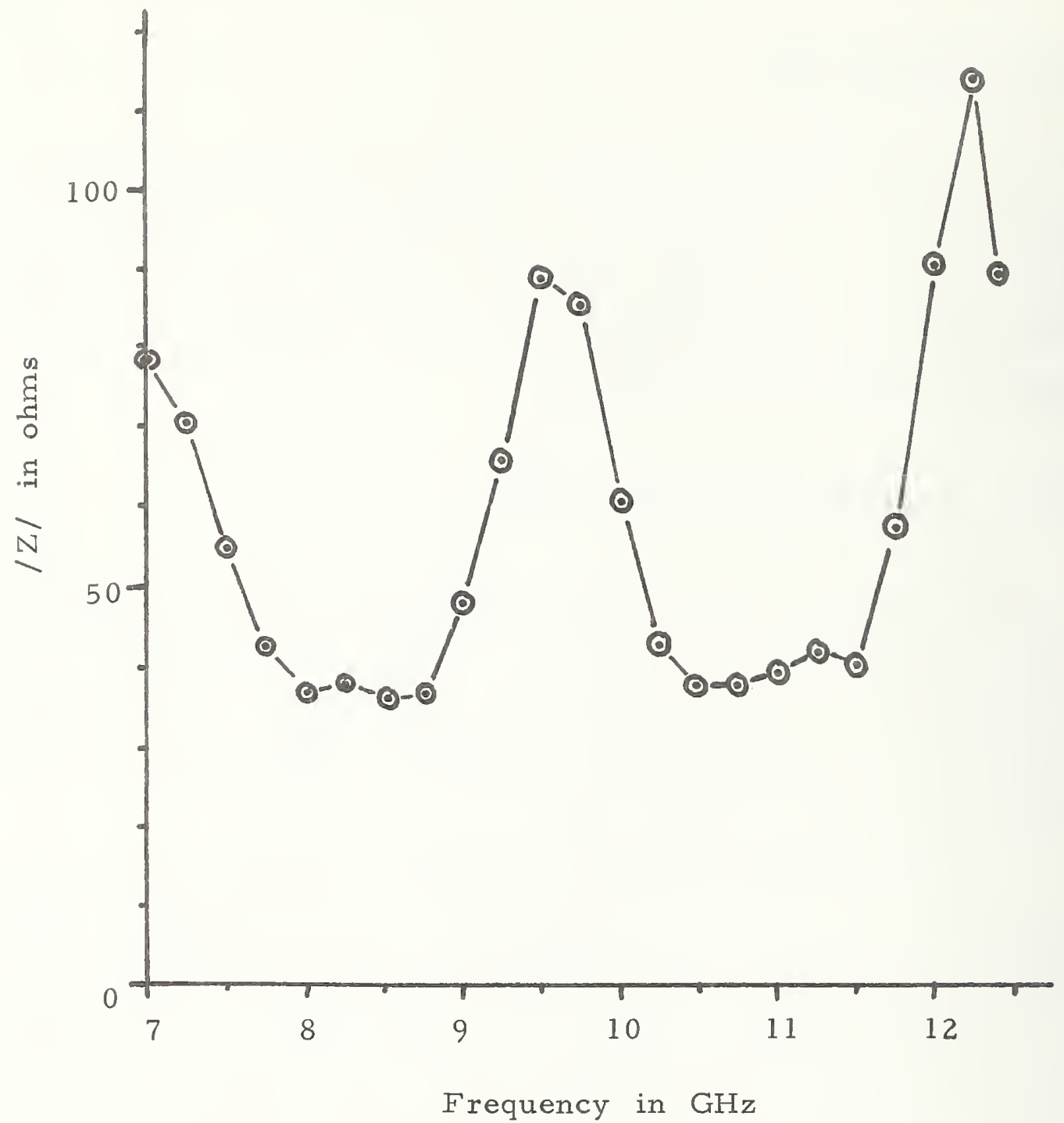

Figure 4.1 Typical results for the magnitude of the input impedance, $|z|$, as a function of frequency. 


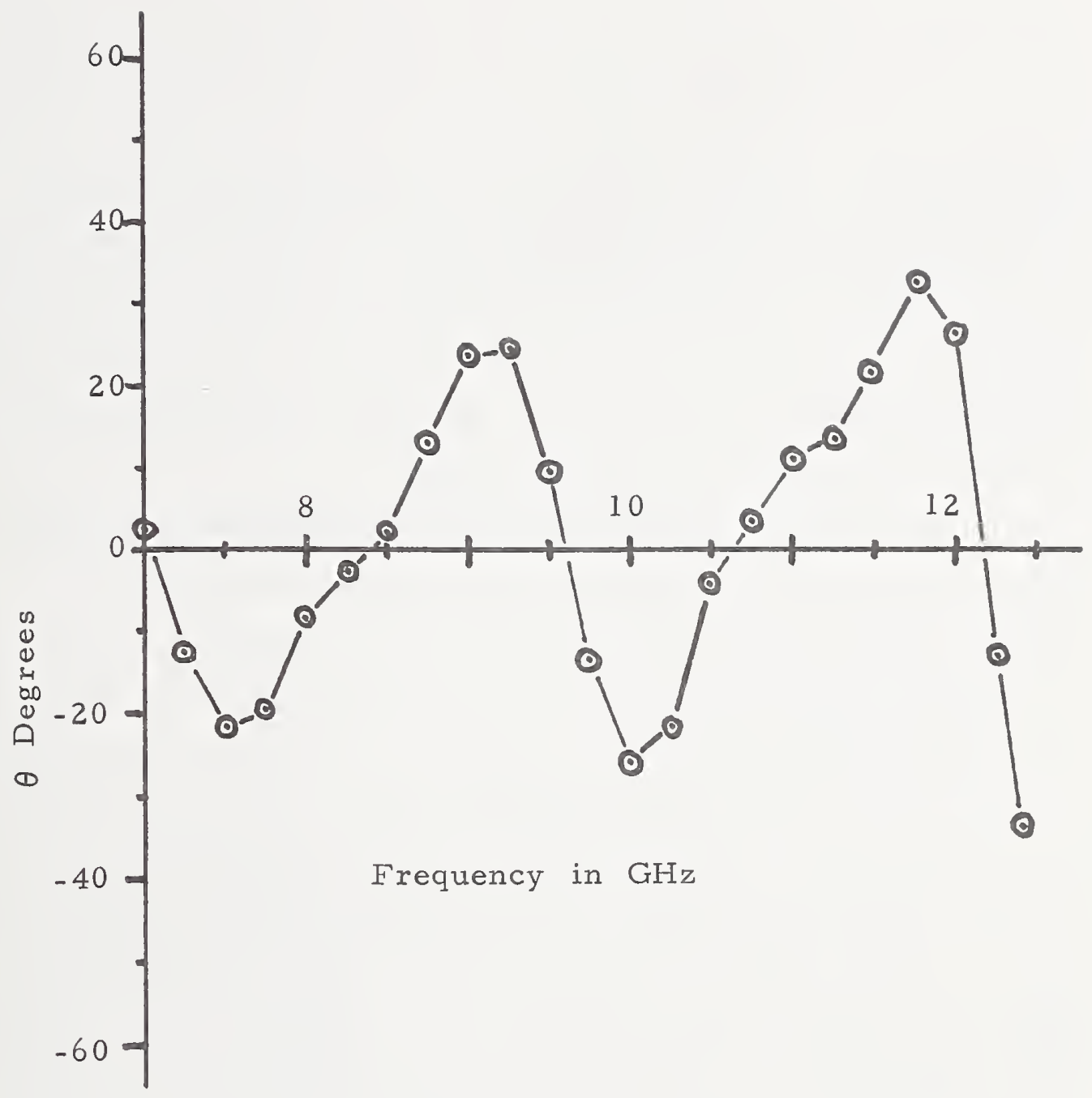

Figure 4.2 Typical results for the phase angle of the input impedance, $\theta$, as a function of frequency. 
from the input plane, the discontinuities arise from four components, Fig. 4.3 ; in order, they are the input connector, the sampling gate, the blow-by compensator probe, and the output connector. The dis continuities are predominately capacitive elements; accordingly, with the transmission line physical lengths the equivalent circuit of Fig. 4. 4 is a reasonable one to assume. As indicated, the transmission line lengths $l_{1}, l_{2}$, and $\ell_{3}$ are $5.1,5.1$, and $5.6 \mathrm{~cm}$, respectively. $Y_{c}$ denotes a connector discontinuity, $\mathrm{Y}_{\mathrm{g}}$ that of the sampling gate, and $Y_{b}$ that of the blowby compensator probe.

Because the magnitudes of the discontinuity admittances are small compared to the characteristic admittance of the 20 millimho coaxial lines which predominately comprise $b_{1}, l_{2}, l_{3}$, the electrical wavelength of the loaded transmission line should be close to that of the physical line length. 5.1 and $5.6 \mathrm{~cm}$ represent free space half-wavelengths corresponding to the frequencies 2.94 and 2.68 $\mathrm{GHz}$, respectively. Consequently, when the sampling-head is terminated in a 20 millimho load, the input admittance (or impedance) should oscillate with a period somewhat less than 2.94 or $2.68 \mathrm{GHz}$ due to the capacitive loading. That such is the case is shown by the data in Figs. 4.1 and 4.2 where the oscillation is about $2.5 \mathrm{GHz}$; also, the presence of a ripple superimposed on the oscillation is consistent with the fact the physical structure is not periodic though multiply loaded. 


\begin{tabular}{|c|c|c|c|c|c|c|}
\hline \multirow{2}{*}{$\begin{array}{c}\text { Input } \\
\text { Connector }\end{array}$} & $\ell_{1}$ & \multirow{2}{*}{$\begin{array}{l}\text { Sampling } \\
\text { Gate }\end{array}$} & \multirow[t]{2}{*}{$\ell_{2}$} & \multirow{2}{*}{$\begin{array}{l}\text { Blow - by } \\
\text { Compensator } \\
\text { Probe }\end{array}$} & $l_{3}$ & \multirow{2}{*}{$\begin{array}{c}\text { Output } \\
\text { Connector }\end{array}$} \\
\hline & & & & & & \\
\hline
\end{tabular}

Figure 4.3 Sampling head physical structure.
Input Port
Output Port
Ref. Plane
Ref. Plane

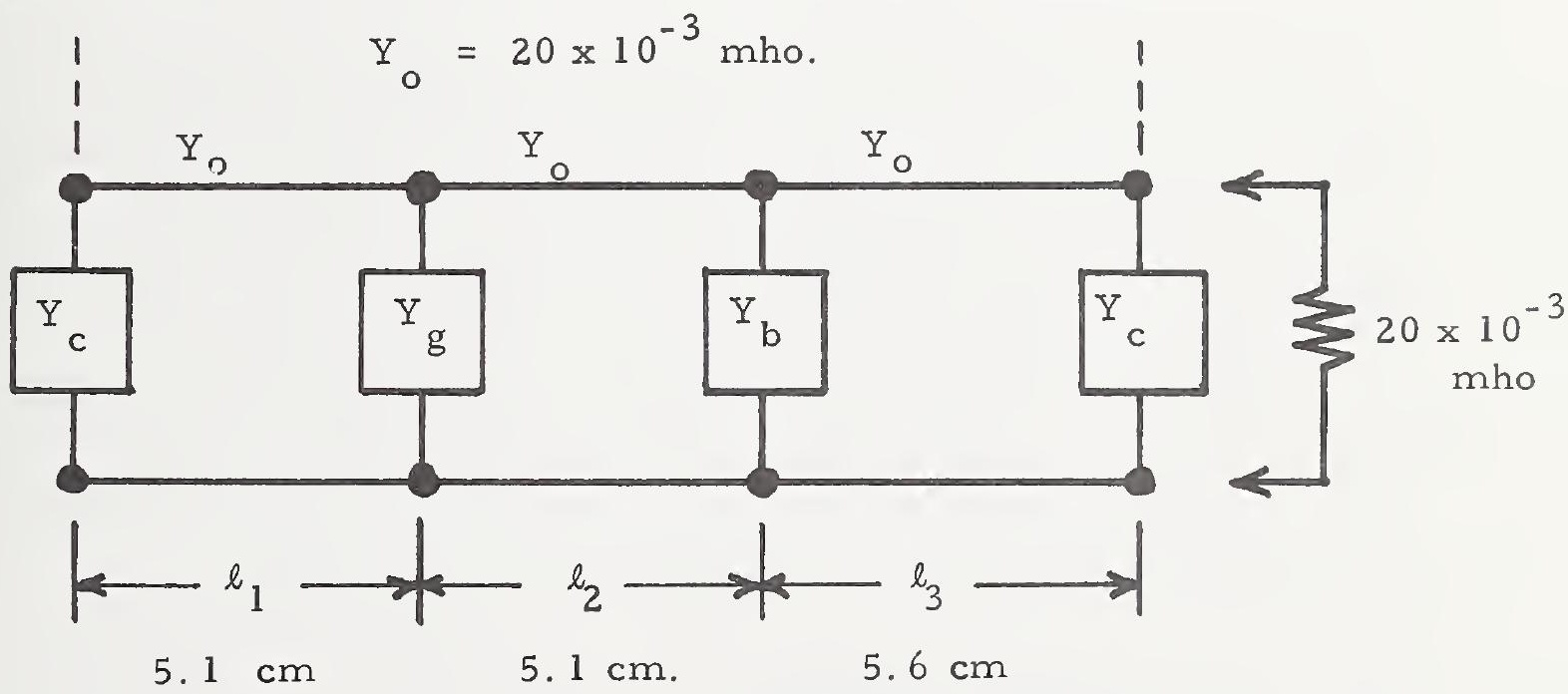

Figure 4.4 Loaded-transmission line equivalent network deduced from the physical structure of the sampling head. 


\section{IMPEDANCE PERTURBATION MEASUREMENTS}

\subsection{Introduction}

In Chapter 2 it was shown that a known impedance perturbation could be used to experimentally determine the turns ratio (2.13) of the transformer in the lossless canonical measurement model. In this chapter typical experimental data will be presented for the observed incremental capacitance due to the insertion of a capacitance perturbation.

5.2 Capacitance Perturbation

Two dummy sampling-diodes were used for the capacitance perturbation experiments. A section of cross-linked polystyrene plastic was machined to the same size as a sampling-diode and tipped with a tinned copper wire imbedded in the plastic. The wire was $0.6 \mathrm{~mm}$ in diameter and $4.5 \mathrm{~mm}$ long and was imbedded in the end of the dummy diode which contacted the inner conductor of the sampling head coaxial line. The wire protruded from the plastic just enough to be in contact with the inner conductor. The resultant dummy diodes were rugged and stable and could be handled without fear of breakage.

\subsection{Experimental Technique}

Low frequency capacitance measurements were used to determine the value of the capacitance perturbation. First, the actual sampling diodes and the output port termination were removed from the samplinghead. The unterminated sampling head appears as a capacitive load at low frequencies. Next, the capacitance without diodes was measured 
and recorded. The dummy diodes were inserted, and the capacitance measurement repeated. The difference between the two observed capacitances was equal to the value of perturbation.

Because the physical dimensions of the sampling-diodes are so small, their loading effect on the signal line is localized to a region much less than a quarter wave up to $18 \mathrm{GHz}$. Consequently, the perturbation capacitance will be independent of frequency up to $18 \mathrm{GHz}$. This in turn means that low frequency measurements may be used to determine the capacitance of the perturbation.

Once the capacitance has been determined, then it may be used as $\Delta$ in the microwave measurements relevant to determining the turns ratio $n,(2.13)$.

\section{5. $4 \quad$ Experimental Results}

Typical experimental results a re shown in Table 5. 1. Low frequency capacitance bridge measurements were made at $1 \mathrm{KHz}$ and $30 \mathrm{MHz}$; introduction of the dummy diodes produced a capacitance change $\Delta \mathrm{C}$ of about $0.035 \pm 0.005 \mathrm{pF}$ at both frequencies. Microwave slotted line measurements at $10 \mathrm{GHz}$ using the perturbation method encompassed in (2-11) and (2-12) gave a $\delta(0.028 \pm 0.005 \mathrm{pF})$ which was essentially equal to $\Delta(0.035 \pm 0.005 \mathrm{pF})$ because the uncertainty was within the attainable accuracy of the experimental measurements. For these results at $10 \mathrm{GHz} \mathrm{n}_{1}=1$ which in turn says that the transformer effects in the canonical network are negligible at $10 \mathrm{GHz}$. 
TABLE 5.1 LOW AND MICROWAVE FREQUENCY MEASUREMENTS OF CAPACITANCE PERTURBATION AT SAMPLING POINT WITHIN THE SAMPLING HEAD

Frequency

Capacitance Perturbation $\Delta C$

$\Delta \mathrm{C} \quad$ Uncertainty

$1 \mathrm{kHz}$

$0.035 \mathrm{pF}$

$\pm 0.002 \mathrm{pF}$

$30 \mathrm{MHz}$

$0.036 \mathrm{pF}$

$\pm 0.005 \mathrm{pF}$

$10 \mathrm{GHz}$

$0.028 \mathrm{pF}$

$\pm 0.005 \mathrm{pF}$ 
6.1 Introduction

The sampling-head consists of a signal transmission line (coaxial line) shunt-loaded at some point by a balanced sampling gate. Qualitatively, if the outer conductor of the coaxial transmission line were pictured as being longitudinally slit and folded out into a plane surface, the sampling head network would appear as a strip line structure, Fig. 6.1. The input signal to the sampling head is $e_{1}(t)$ while the balanced samplingpulse is $\pm e_{S}(t-\tau)$ which drives the sampling gate. The diodes $D_{1}$ and $\mathrm{D}_{2}$ comprise the sampling gate and are hot carrier diodes which are normally biased to cutoff. When the sampling pulse occurs at $t=\tau_{1}$ the diodes conduct strongly and provide two equal paths for signal currents from the signal line. Each path essentially consists of a diode in series with a sampling (storage) capacitor and a sampling pulse generator. Ideally, the sampling path or current loop should be such that the loop current $i_{s}(t)$ should have a transition time of zero, i. e., it should jump to its final value in zero time at the instant that the loop switches are closed. In practice, the impedance of the sampling current loop is not purely resistive; consequently, the current transition time will not be zero. If the impedance of the conducting diodes and all other elements appearing in the sampling-loop are lumped together into a single impedance $Z_{s}(s)$, the sampling loop can be represented as shown in Fig. 6.2A where the diode gating or switching function is represented by two synchronized ideal switches. When the switches are closed, the equivalent circuit of Fig. 6.2B results.

Assuming that the signal line is lossless, then the measurement methods described in Section 2.6 may be applied so that the sampling loop impedance may be observed by slotted-line impedance measurements. 


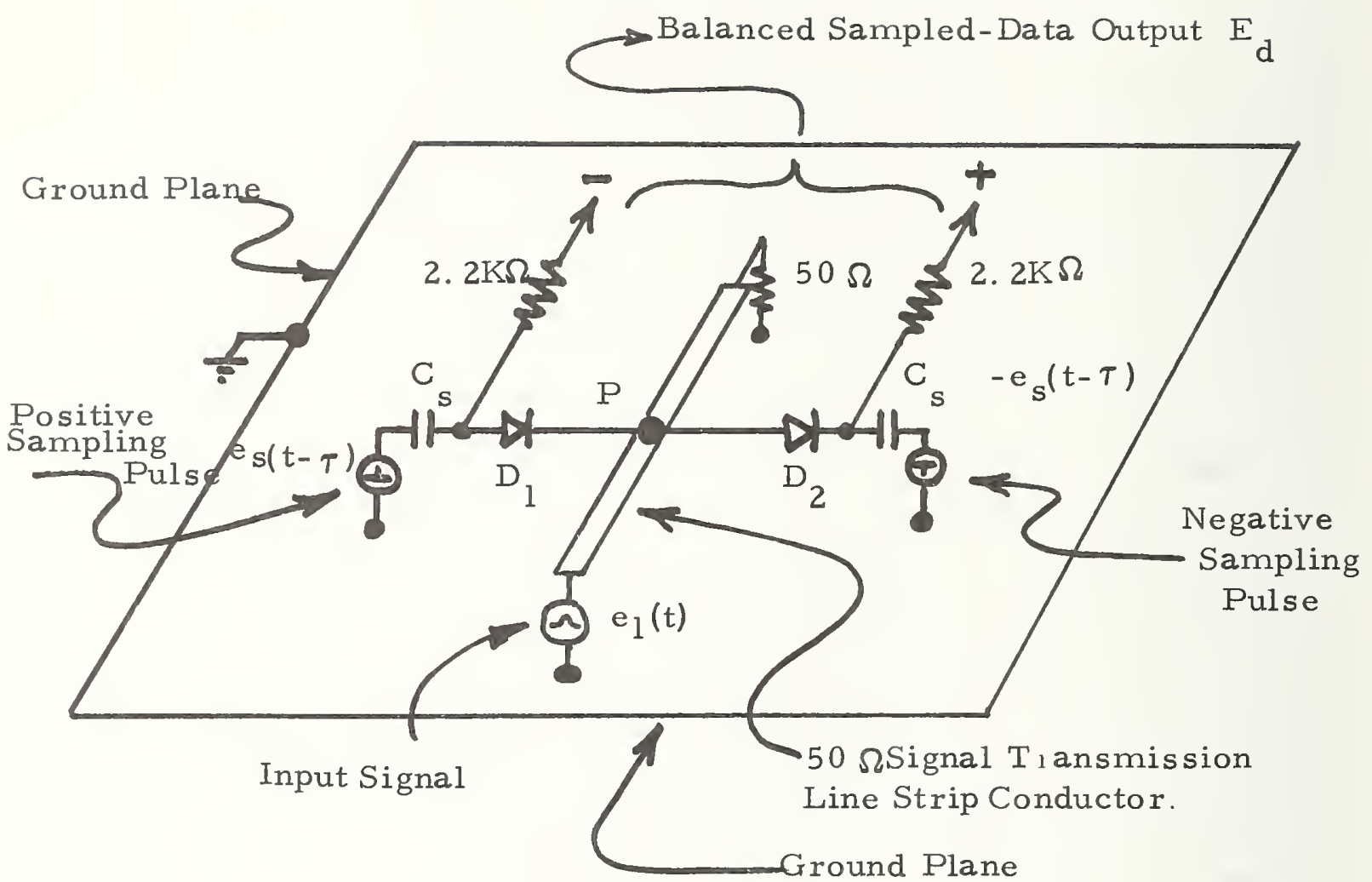

Figure 6.1 Balanced sampling gate across an unbalanced signal transmission line represented in a strip line geometry. 


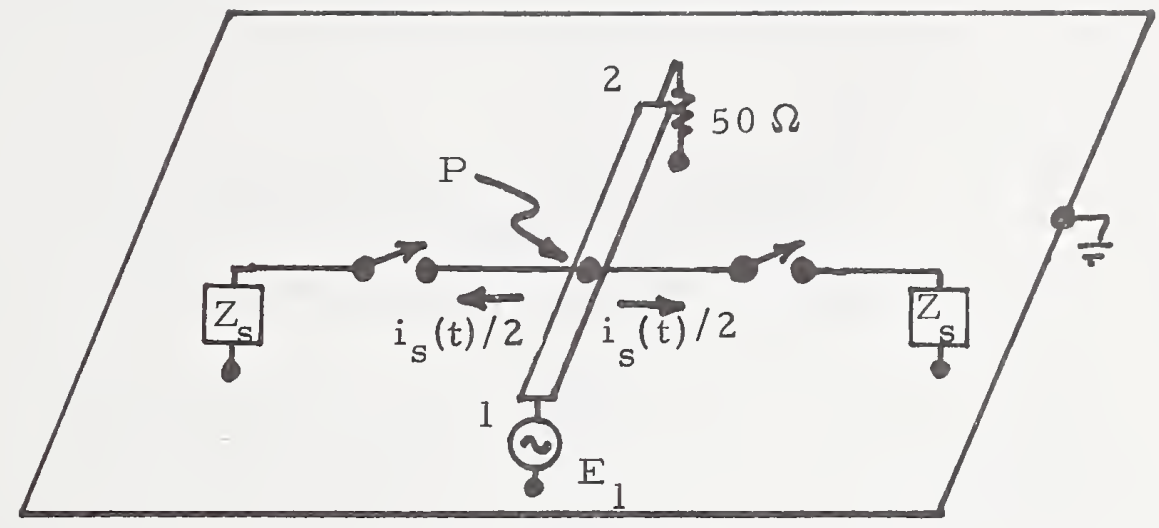

Figure 6.2 (A) Sampling loop with loop switches open.

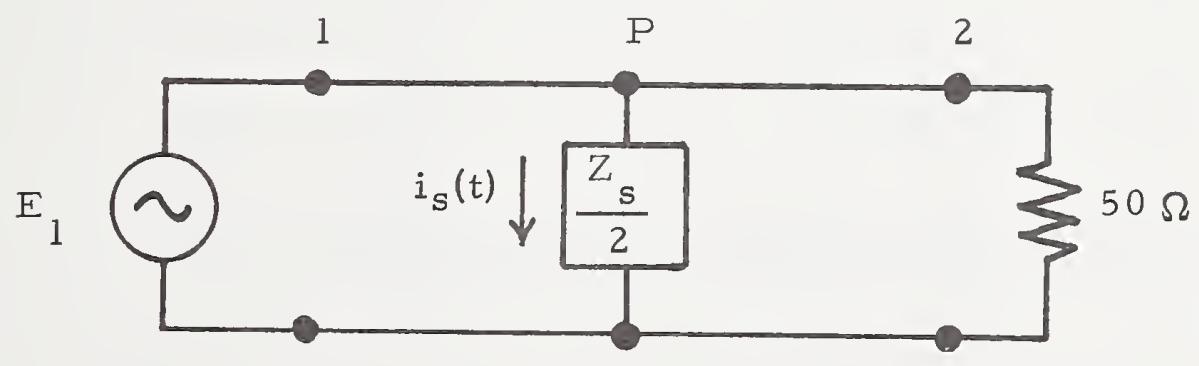

Figure 6.2 (B) Equivalent circuit when sampling loop switches are closed. 
Fig. 6.3 shows the equivalent circuit reduction for the measurement method. Briefly, the position of the sliding short on port 2 is adjusted to be $\lambda / 4$ or $(2 N-1) \lambda / 4$ from sampling point $P$, thus presenting an open circuit to the right of $P$. If the position of the slotted line probe, point 1 , is adjusted to be $\lambda / 2$ or $N \lambda / 2$ then the impedance at 1 is the same as at $P$. Fig. 6. 4 gives an equivalent circuit for the sampling loop $[5,6]$. $R_{j}$ and $C_{j}$ are the resistance and capacitance of a diode junction, respectively; they are equivalent circuit elements for the diode depletion region electronic phenomena and are functions of the diode dc bias current, $I_{b}$. $C_{s}$ is a sampling capacitor which stores the sampled charge. $R$ is principally due to the bulk resistivity of the metals and semi-conductors on each side of the depletion region in a diode; it also includes the resistance of the sampling loop conductors. L is principally due to the diode catwhisker conductor but also includes the inductance of the sampling loop conductors. $\mathrm{C}$ is the package capacitance which physically bridges the diode terminals. Fig. 6.5 shows the physical structure of a sampling diode package. Note that $\mathrm{C}_{\mathrm{S}}$ is built into the package. Finally, when the diodes are conducting, the two diode-sampling paths a re in parallel; hence, the overall equivalent circuit contains the individual equivalent circuit parameters multiplied or divided by a factor of two.

\subsection{Sampling-Loop Impedance vs. Bias Current}

At this point it is instructive to qualitatively discuss the behavior of the sampling-loop impedance as the bias current is varied. Typically, when the bias current is zero, $R_{j}$ is very large and the diode is cut off. The sampling loop impedance $Z_{s}(s) / 2$, Fig. 6.4, is primarily a capacitive reactance. When the bias current is applied $R_{j}$ is small and a high frequency signal current flows through $\mathrm{R} / 2, \mathrm{~L} / 2$ and the diode junction impedance. For a large enough bias current, most of the signal current flows through the diode branch, and the impedance appears to be inductive. 

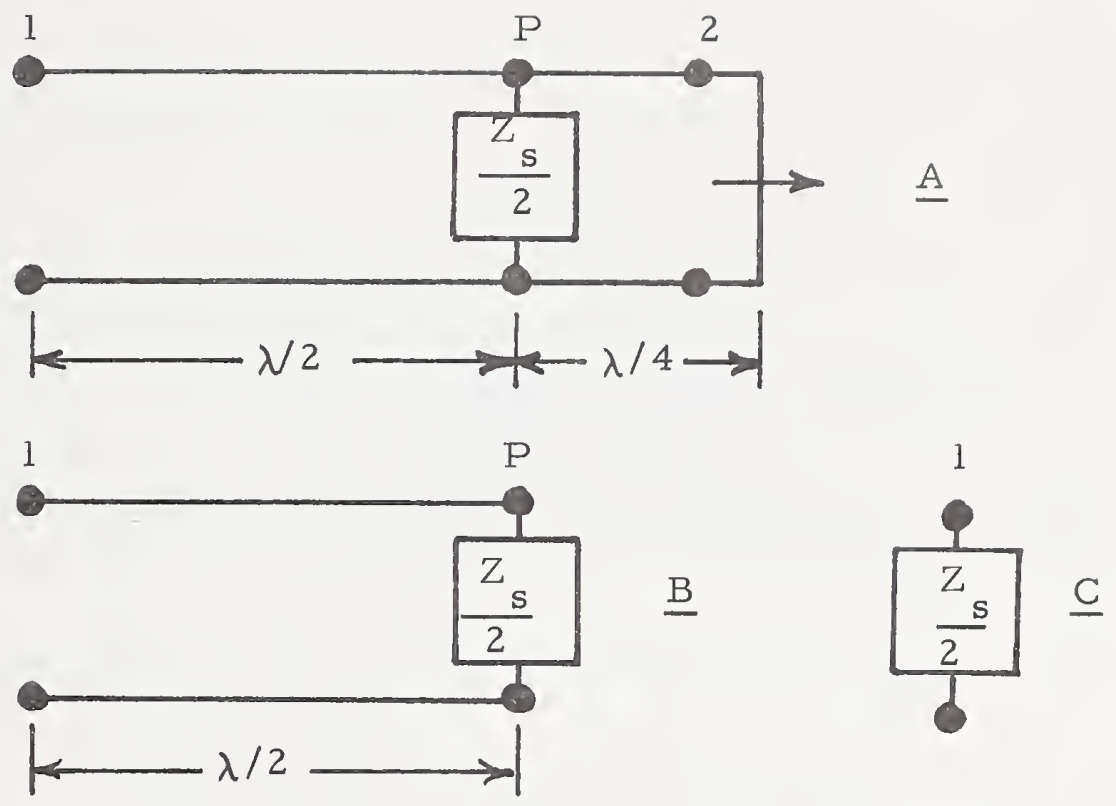

Figure 6.3 Equivalent circuit reduction for the measurement method of Section 2.6 applied to the measurement of the sampling loop impedance $Z_{s}(s) / 2$.

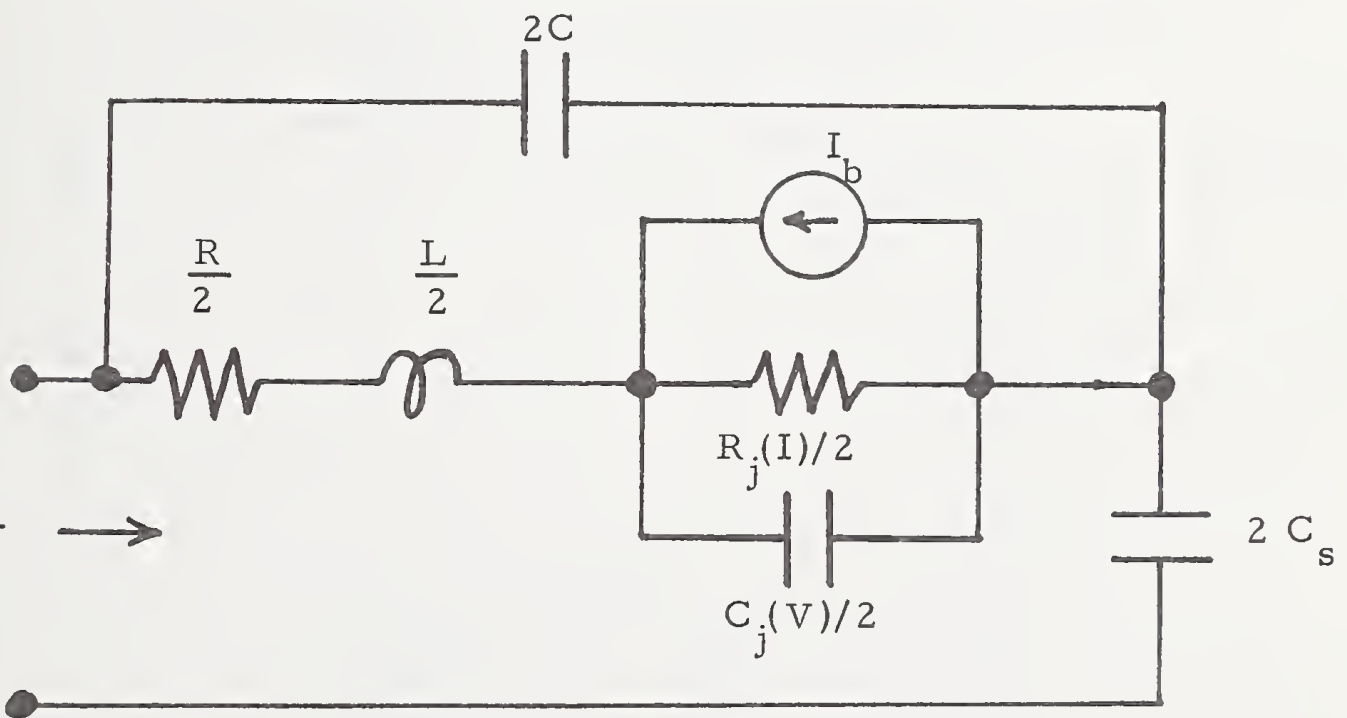

Figure 6.4 Equivalent circuit for the sampling current loop. 

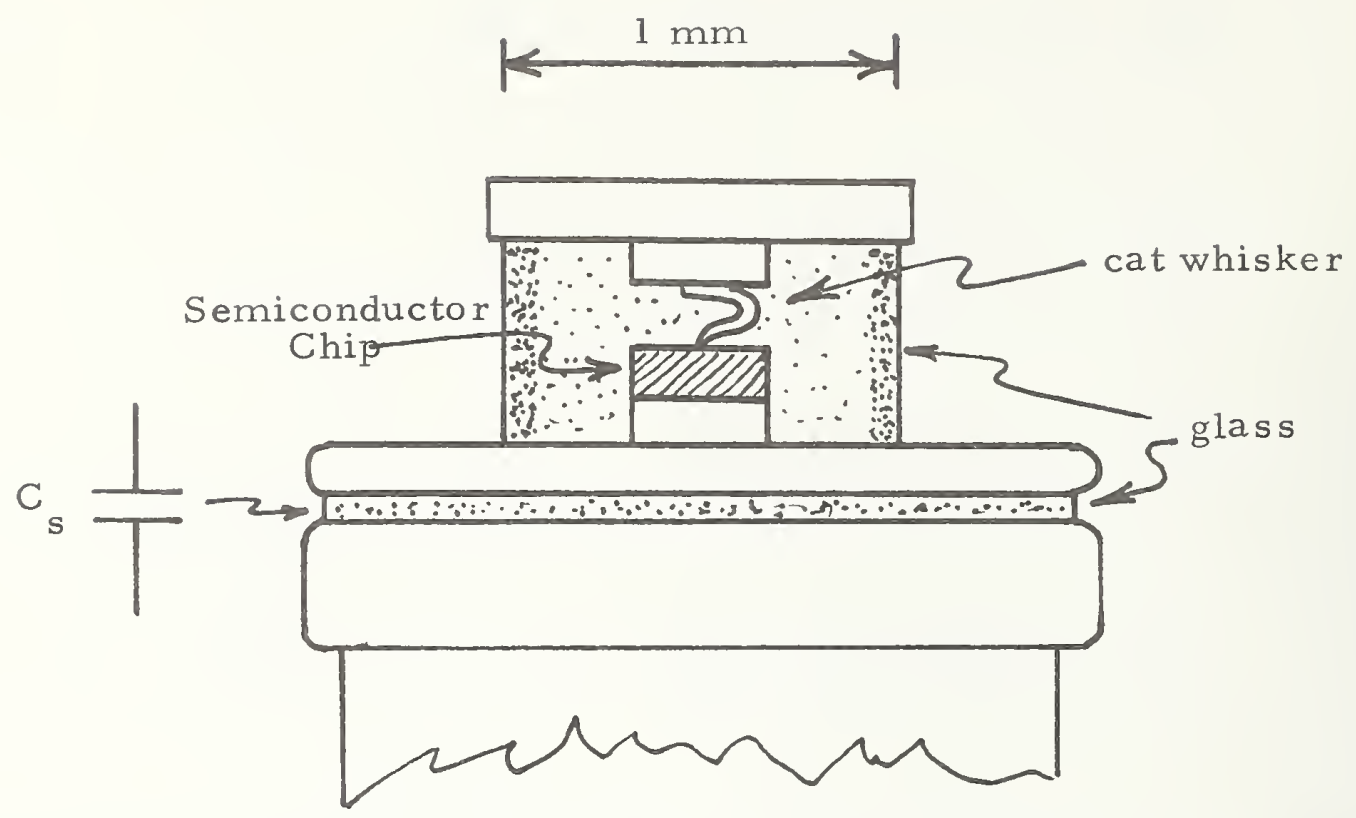

Figure 6.5 Physical structure of the sampling diode package.

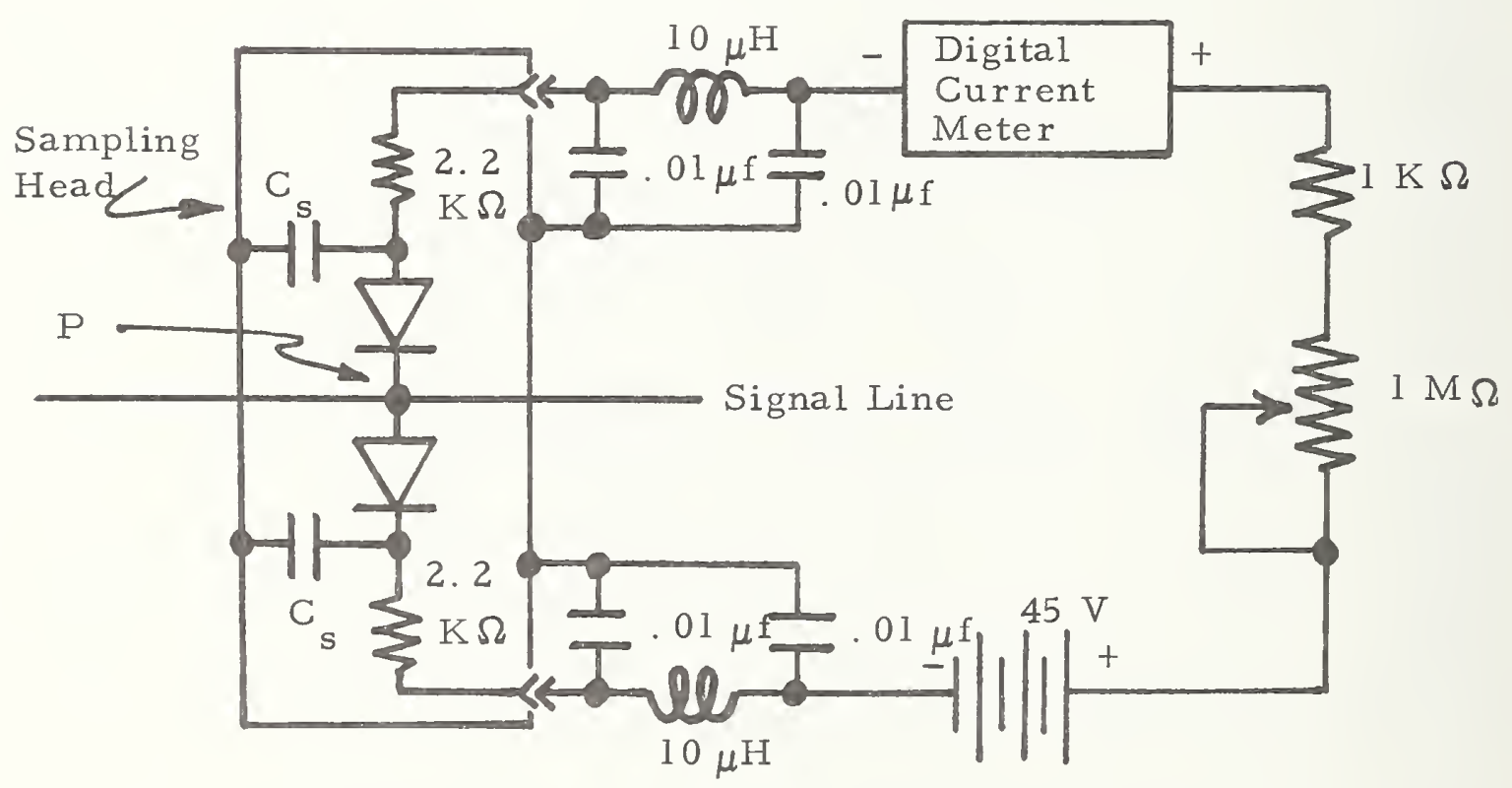

Figure 6.6 External bias supply connected to the diode sampling gate. 
By measuring the sampling loop impedance as a function of frequency and bias current, it is possible to deduce the element values for the equivalent circuit of Fig. 6.4. In the present study, impedance measurements were made at one frequency, $10 \mathrm{GHz}$, over a bias range of 0 to $10 \mathrm{ma}$. The impedance varied from about $65-\mathrm{j} 95$ to $14.5+\mathrm{j} 5$ ohms. The $10 \mathrm{GHz}$ resistance seen for 5 ma bias was equal to the $5 \mathrm{ma}$ dc dynamic resistance of the sampling diode. Thus, it appeared that at $10 \mathrm{GHz}$ the hot carrier diodes were still functioning efficiently.

\subsection{Experimental Impedance Measurements and Results}

Using the microwave impedance measurement system shown in Fig. 2.7, the impedance measurement reference planes a re established by the procedure described in Section 2.6. The $50 \mathrm{ohm}$ termination on the sampling head is replaced by a sliding short. The oscilloscope is then shut-off, and an external biasing supply connected to the diode samplinggate, Fig. 6.6. Slotted-line impedance measurements a re then made as a function of the sampling-gate bias current.

Figs. 6.7 and 6.8 present typical $10 \mathrm{GHz}$ data for the real part of the sampling-loop impedance $R_{S}$ and the imaginary part of the samplingloop impedance $X_{s}$, each as a function of the sampling bias current, $I_{b}$.

When the sampling-diodes a re conducting strongly $\left(I_{b}=5 \mathrm{ma}\right)$, Fig. 6.7 shows a $10 \mathrm{GHz}$ resistance of $14.5 \mathrm{ohms}$ for the two diodes in parallel. This value correlates very well with the slope of the static I-V curve for the sampling diodes. Fig. 6.9 shows the static I-V curve for one sampling diode. At $I=5 \mathrm{ma}$ the slope $\Delta \mathrm{V} / \Delta \mathrm{I}$ equals 28.5 ohms. For two diodes in parallel the value would be 14.25 ohms which is very close to the $10 \mathrm{GHz}$ value. Because the diodes are hot carrier devices, it is expected that their conduction process should be relatively independent of frequency to well above $20 \mathrm{GHz}$. 
Fig. 6.8 shows a $10 \mathrm{GHz}$ reactance of +5 ohms at $I_{b}=5 \mathrm{ma}$ Referring to Fig. 6. 4, if the sampling-capacitor's reactance, $\omega 2 \mathrm{C}_{\mathrm{s}}$, is negligible and the current all flows through the inductive branch, then the observed inductive reactance should be $\sim \omega L / 2$. Typically $2 \mathrm{C}_{\mathrm{s}}$ is $4 \mathrm{pF}$, consequently at $10 \mathrm{GHz}$ the sampling capacitor reactance would be about $-0.4 \mathrm{ohms}$; accordingly, the observed reactance would be $-0.4+\omega L / 2$, and $\omega L / 2$ would equal $5.4 \mathrm{ohms}$. This in turn would give an $\mathrm{L}$ of about $170 \mathrm{pH}$ which is of the same order as reported earlier $[5,6]$. 


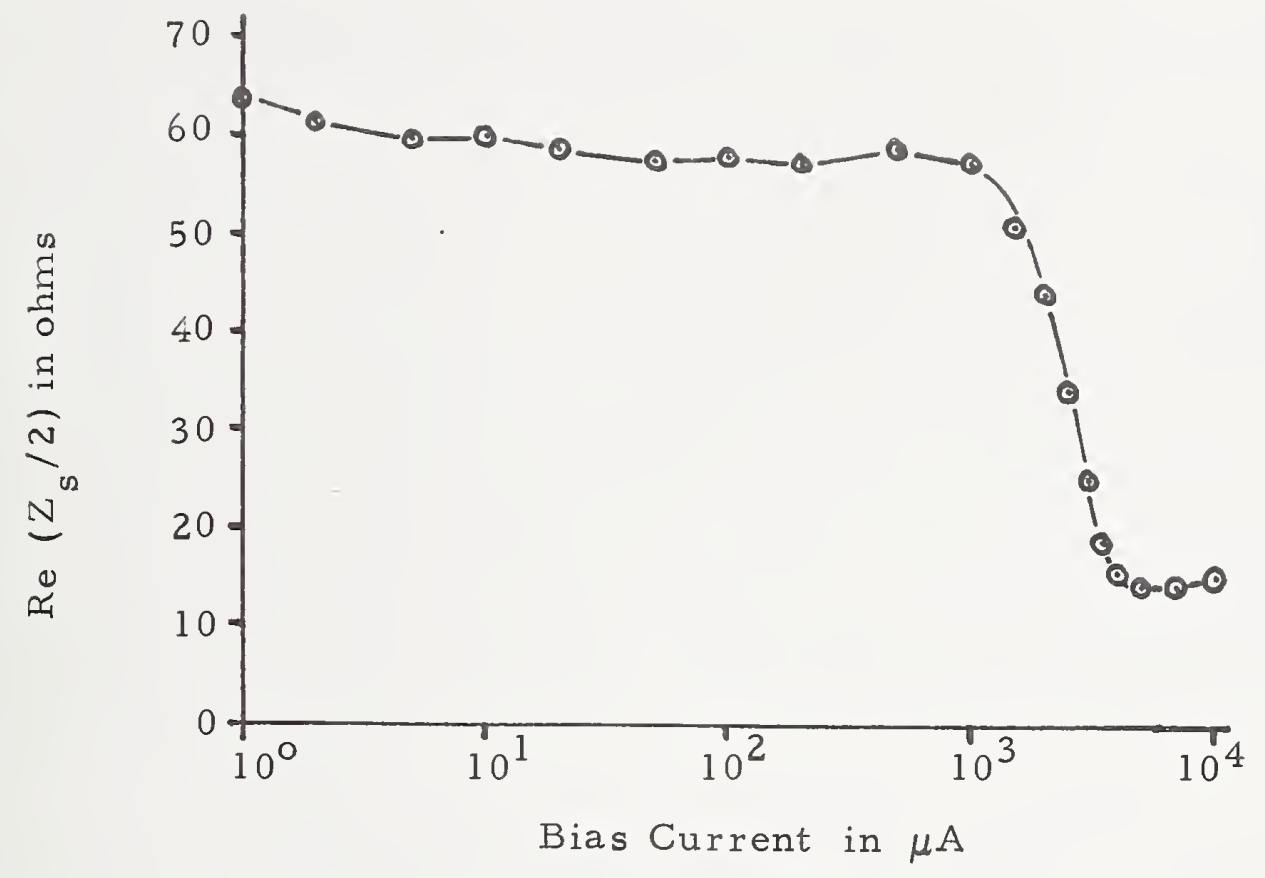

Figure 6.7 Real part of the $10 \mathrm{GHz}$ sampling loop impedance vs. sampling diode bias current.

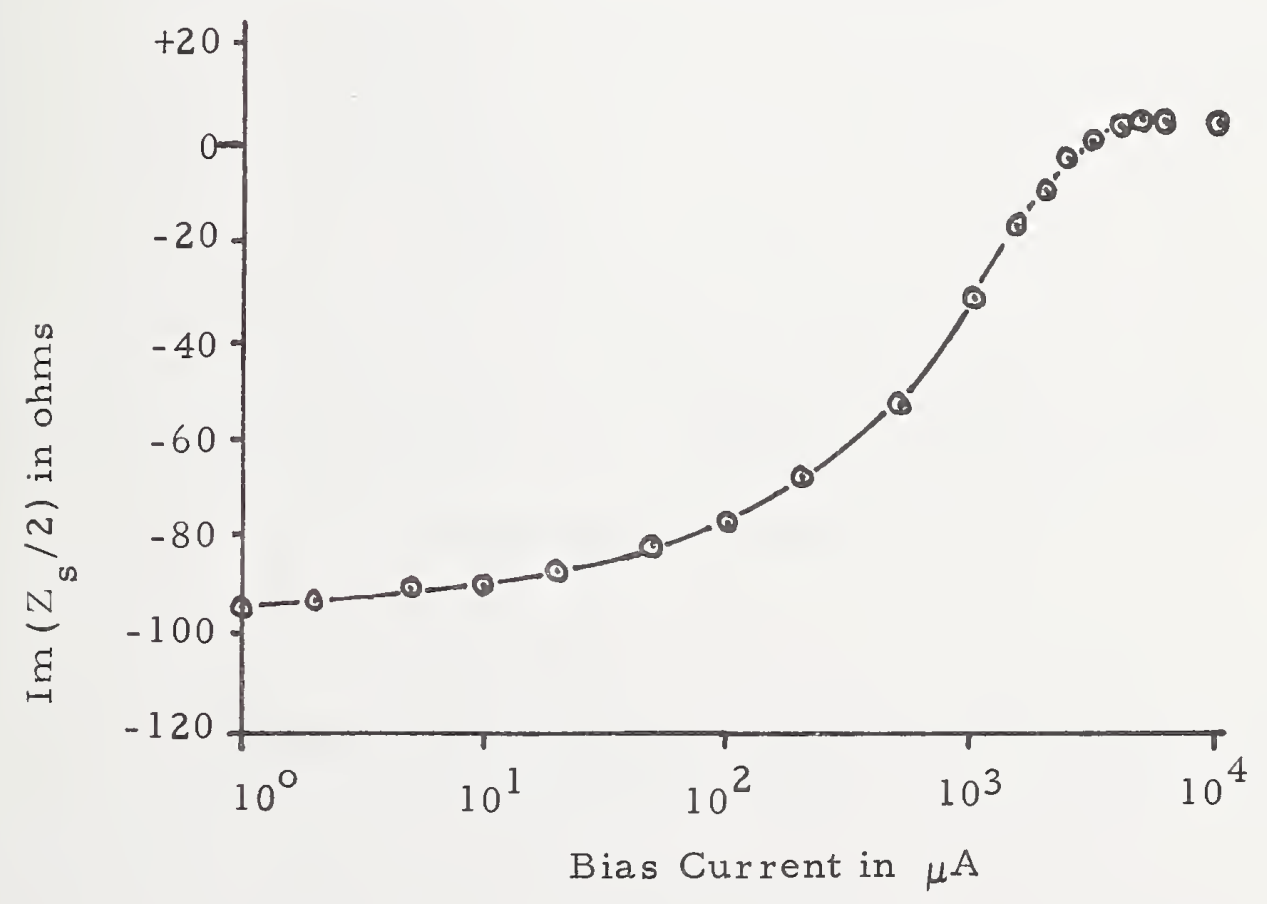

Figure 6.8 Imaginary part of the $10 \mathrm{GHz}$ sampling loop impedance vs. sampling diode bias current. 


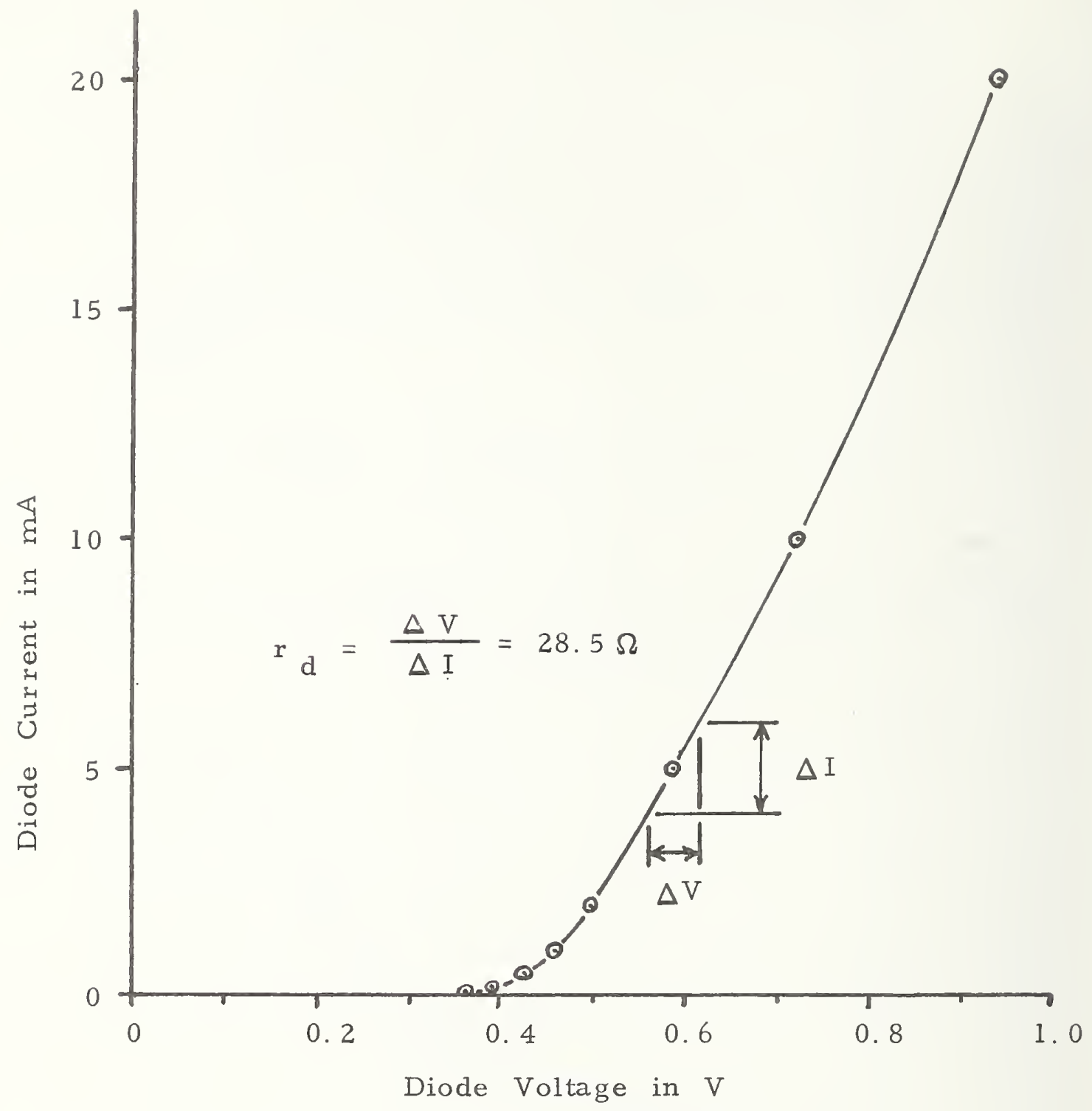

Figure 6.9 Static I-V characteristic for a sampling diode. 
7. SUMMARY AND RECOMMENDATIONS FOR FUTURE WORK

\subsection{Summary}

The major results of this work have been the development of microwave voltage and impedance measurement techniques which provide the means for determining the equivalent circuit parameters for the sampling head when applied over suitable frequency ranges. The techniques are based upon slotted-line measurements and are not inherently limited to any particular frequency range. Experimental data were obtained in the 7 to $12.4 \mathrm{GHz}$ range.

\subsection{Recommendations for Future Work}

Additional work should proceed in two phases:

1. Coaxial slotted-line measurements should be extended up to $18 \mathrm{GHz}$; such measurements would require the use of the lossy canonical form measurement network. However, the reduced data would provide additional information about the sampling-loop impedance. This in turn would establish the sampling-gate equivalent network and in conjunction with sampling pulse shape estimates allow an extrapolation to estimate higher frequency performance (above $18 \mathrm{GHz}$ ).

2. Waveguide slotted-line measurements should be applied over the 18 to $26.5 \mathrm{GHz}$ band; such measurements would provide data on the frequency response cut-off characteristics of the sampling head due to sampling-gate frequency limitations and transmission line multimode effects. 
Results would provide an examination of the effectiveness of the extrapolation methods in the first phase and serve as a basis for further extrapolation to the $40 \mathrm{GHz}$ frequency range. 
[1] Ginzton, E. L., Microwave Measurements, McGraw-Hill, Inc., New York, 1957, pp. 323-326.

[2] Ginzton, E. L., ibid., pp. 343-345.

[3] Ginzton, E. L., ibid., pp. 277-283.

[4] Ginzton, E. L., ibid., pp. 274, 278-285.

[5] Howard, D., Best, A., Humphrey, J., "The Wideband Sampling Gate, an Analysis, Characterization and Discussion". WESCON Proceedings, Session 23, Aug. 23-26, 1966, Paper 23/1.

[6] Grove, Wayne, "Sampling for Oscilloscopes and Other R. F. Systems: DC through X-band". IEEE Trans. Microwave Theory and Techniques, Vol. MTT-14, No. 12, pp. 629-635,

December 1966.

[7] Johnson, Walter C., Transmission Lines and Networks, McGraw-Hill, N. Y., 1950, chp. 4, in particular see p. 100.

[8] Nahman, N. S., "A Discussion on the Transient Analysis of Coaxial Cables Considering High-Frequency Losses", IRE Trans. Professional Group on Circuit Theory, Vol. CT-9, No. 2, June 1962, pp. 144-152. 


\section{APPENDIX}

THE ANALYSIS OF DISCRETELY LOADED TRANSMISSION LINES IN TERMS OF TRANSMITTED AND REFLECTED PULSE TRAINS

In the classical frequency domain analysis of transmission lines containing reflections, the as sumption is implicit that the sinusoidal signals have been present since minus infinity. In time domain instruments for the measurement of transient phenomena, such as a sampling oscilloscope, such is not the case. Prior to some time, say $t=0$, there was no signal present. When the signal does enter the transmission line it propagates along the line at a finite velocity arriving at observation points displaced from the input later in time. When this propagating signal encounters a discontinuity reflected and transmitted signals are created. An observation point remote from the point of reflection will not observe the reflected signal until still later in time due to the time isolation (delay) inherent in the transmission line. For people working with time domain instruments it is very important to comprehend this time isolation of reflections. As an aid to developing an understanding of this concept this appendix develops the analysis of discretely loaded transmission line in terms of transmitted and reflected pulse trains. A reflection-transmission ( $R T$ ) diagram is used. The resultant transmission line equations are then shown to be identical to those derived in the classical frequency domain manner. 
In general, the generator to transmission line terminals transfer functions, Fig. A-1, can be written [7] as

$\frac{E_{s}(s)}{E_{g}(s)}=\left[\frac{Z_{0}(s)}{Z_{s}(s)+Z_{0}(s)}\right]\left[\frac{1}{1-\rho_{s}(s) \rho_{r}(s) e^{-2 l \gamma(s)}}\right]\left[1+\rho_{r}(s) e^{-2 l \gamma(s)}\right]$

and

$\frac{E_{r}(s)}{E_{g}(s)}=2\left[\frac{Z_{o}(s)}{Z_{s}(s)+Z_{o}(s)}\right]\left[\frac{Z_{r}(s)}{Z_{o}(s)+Z_{r}(s)}\right]\left[\frac{e^{-l \gamma(s)}}{1-\rho_{s}(s) \rho_{r}(s) e^{-2 l \gamma(s)}}\right]$

where $Z_{s}(s)$ and $Z_{r}(s)$ are the sending and receiving end impedances, respectively. ${ }^{*} \rho_{s}(s)$ and $\rho_{r}(s)$ are the sending and receiving reflection coefficients, respectively.

$$
\begin{aligned}
& \rho_{s}(s)=\frac{Z_{s}(s)-Z_{0}(s)}{Z_{s}(s)+Z_{0}(s)} \\
& \rho_{r}(s)=\frac{Z_{r}(s)-Z_{o}(s)}{Z_{r}(s)+Z_{o}(s)}
\end{aligned}
$$

*To obtain equation A-2 from Johnson's [7] equation 4.23 the relationship

$$
1+\rho_{r}(s)=\frac{2 Z_{r}(s)}{Z_{0}(s)+Z_{r}(s)}
$$

is used. 


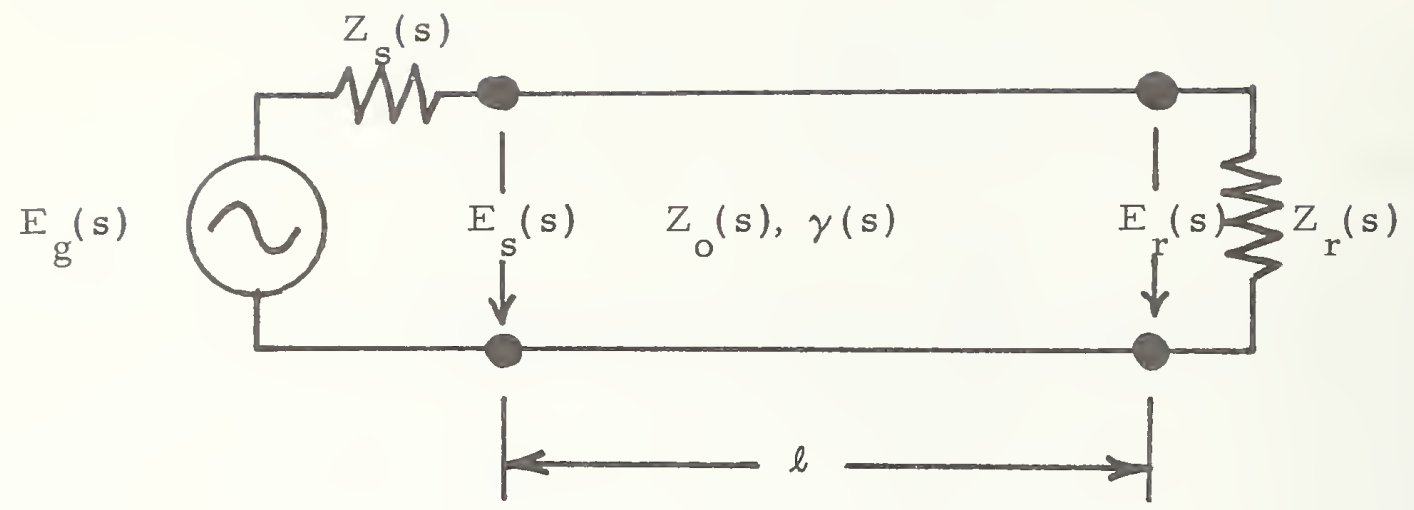

Figure A-1 Uniform transmission line connecting an arbitrary generator and arbitrary load.
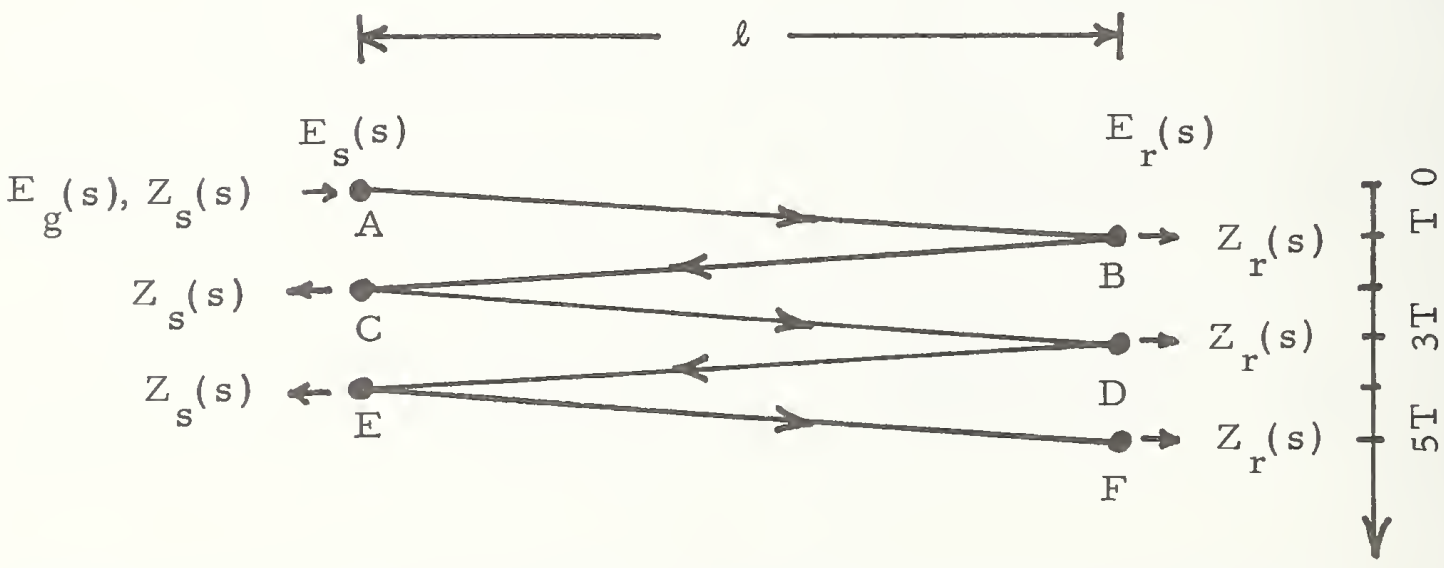

Figure A-2 The RT diagram for a uniform transmission line connecting an arbitrary generator and load. 
$l$ is the transmission line length while $\gamma(s)$ and $Z_{0}(s)$ are the line propagation function and characteristic impedance, respectively.

$$
\begin{aligned}
& \gamma(s)=[Z(s) Y(s)]^{1 / 2} \\
& Z_{0}(s)=\left[\frac{Z(s)}{Y(s)}\right]^{1 / 2}
\end{aligned}
$$

$Z(s)$ and $Y(s)$ are the transmission line equivalent circuit parameters: series impedance/meter and shunt admittance/meter. $\gamma(s)$ is of the form $s T^{\prime}+\gamma^{\prime}(s)$, where $T^{\prime}$ is the constant $\sqrt{\mathrm{LC}}$; the time delay per unit length (see discussion following equation A-16).

Now consider a step generator of impedance $Z_{0}(s)$ applied to the system described by $(A-1)$ and $(A-2)$, i. e., $E_{g}(s)=1 / s$ and $Z_{s}(s)=Z_{0}(s)$. Then $\rho_{s}(s)=0$, and $(A-1)$ and $(A-2)$ reduce to

$$
E_{s}(s)=\frac{1}{2 s}\left[1+\rho_{r}(s) e^{-2 \ell\left[s T^{\prime}+\gamma^{\prime}(s)\right]}\right]
$$

and

$$
E_{r}(s)=\frac{1}{s}\left[\frac{Z_{r}(s)}{Z_{0}(s)+Z_{r}(s)}\right] e^{-l\left[s T^{\prime}+\gamma^{\prime}(s)\right]}
$$

Rewriting,

$$
E_{s}(s)=\frac{1}{2 s}+e^{-2 s l T^{\prime}}\left[\rho_{r}(s) e^{-2 \ell \gamma^{\prime}(s)}\right]
$$

and

$$
E_{r}(s)=e^{-s l T^{\prime}}\left\{\frac{Z_{r}(s) e^{-l \gamma^{\prime}(s)}}{s\left[Z_{o}(s)+Z_{r}(s)\right]}\right\}
$$


Consideration of $(A-9)$ and $(A-10)$ leads to the following conclusions:

1. The input terminals of the line presents to the generator the characteristic impedance $Z_{o}(s)$ for the time interval $0<t<2 \ell \mathrm{T}^{\prime} ;$ i. e., the load impedance is not seen at the generator terminals until the incident wave travels to the load and the subsequent reflection returns to the generator terminals.

2. The output waveform is zero for $t<\ell \mathrm{T}^{\prime} ;$ also, its shape is controlled by the characteristic impedance to load impedance voltage divider and the dispersive part of the propagation function, $\gamma^{\prime}(\mathrm{s})$.

Generally, for arbitrary terminations, $E_{S}(s)$ and $E_{r}(s)$ each consist of an infinite sequence of terms similar to those in (A-9) and $(A-10)$ and having their origins in the conclusions delineated above. By using the geometric series expansion

$$
\begin{gathered}
{\left[1-\rho_{s}(s) \rho_{r}(s) e^{-2 \ell \gamma(s)}\right]^{-1}=\sum_{n=0}^{\infty}\left[\rho_{s}(s) \rho_{r}(s) e^{-2 l \gamma(s)}\right]^{n} ;} \\
\left|\rho_{s}(s) \rho_{r}(s) e^{-2 l \gamma(s)}\right|^{2}<1
\end{gathered}
$$

in the second and third factors of $(A-1)$ and $(A-2)$, respectively, the transfer functions can be expressed as infinite sequences which represent impulse responses consisting of pulse trains. Upon putting $(A-11)$ into $(A-1)$ and $(A-2)$ obtains the sending end pulse train 


$$
\begin{aligned}
E_{s}(s) & =E_{g}(s)\left[\frac{Z_{0}(s)}{Z_{s}(s)+Z_{0}(s)}\right] \\
& x \sum_{n=0}^{\infty} \rho_{s}^{n}(s) \rho_{r}^{n}(s) e^{-2 n l} \gamma(s)+\rho_{s}^{n}(s) \rho_{r}^{(n+1)}(s) e^{-(n+1) 2 l \gamma(s)}
\end{aligned}
$$

and the receiving end pulse train,

$$
\begin{aligned}
E_{r}(s) & =2 E_{g}(s)\left[\frac{Z_{0}(s)}{Z_{s}(s)+Z_{0}(s)}\right]\left[\frac{Z_{r}(s)}{Z_{r}(s)+Z_{0}(s)}\right] \\
& X \sum_{n=0}^{\infty} \rho_{s}^{n}(s) \rho_{r}^{n}(s) e^{-(2 n+1) \& \gamma(s)}
\end{aligned}
$$

where $(A-12)$ and $(A-13)$ are both subject to the condition specified in (A-11). The physical situation represented by the pulse trains $(A-12)$ and $(A-13)$ is lucidly shown by a reflection-transmission ( $R T$ ) diagram, Fig. A-2. In fact, the RT diagram, itself, provides an intuitive (but exact) means for writing out the terms of each pulse train.

The R I diagram is a signal flow chart embodying the reflection and transmission properties of the terminal impedances, and the wave propagation property of the connecting transmission line. To interpret the diagram the following rules are used:

1. Passage from a generator of impedance $\mathrm{Z}_{\mathrm{s}}(\mathrm{s})$ through a node to a line segment represents multiplication by the transfer function $Z_{0}(s) /\left[Z_{s}(s)+Z_{0}(s)\right]$.

2. Passage along a line segment represents multiplication by the transfer function $\exp [-\ell \gamma(\mathrm{s})]$. 
3. Passage through a node to a load $Z_{r}(s)$ represents multiplication by the transfer function $2 Z_{r}(s) /\left[Z_{r}(s)+Z_{0}(s)\right]$.

4. Reflection from a node represents multiplication by the reflection coefficient $\rho_{r}(s)=\left[Z_{r}(s)-Z_{o}(s)\right]\left[Z_{r}(s)+\right.$ $\left.\mathrm{Z}_{\mathrm{o}}(\mathrm{s})\right]^{-1}$.

Application of the rules to the diagram in Fig. A-2 obtains the following results:

$$
\begin{aligned}
& E_{s}(s)=\left[E_{g} \frac{Z_{0}}{Z_{s}+Z_{0}}\right]+\left[E_{g} \frac{Z_{0}}{Z_{s}+Z_{0}} e^{-l \gamma} \rho_{r} e^{-l \gamma} \frac{2 Z_{s}}{Z_{s}+Z_{0}}\right] \\
& \text { Gen to } A \\
& A B C \\
& +\left[E_{g}(s) \frac{Z_{o}}{Z_{0}+Z_{s}} e^{-l \gamma_{\rho_{r}}} e^{-l \gamma} \rho_{s} e^{-l \gamma} \rho_{r} e^{-l \gamma} \frac{2 Z_{s}}{Z_{s}+Z_{o}}\right]+\ldots \\
& =E_{g} \frac{Z_{0}}{Z_{s}+Z_{0}}\left\{1+\frac{2 \rho_{r} Z_{s}}{Z_{s}+Z_{0}} e^{-2} \ell \gamma+\frac{2 \rho_{r}^{2} \rho_{s} Z_{s}}{Z_{s}+Z_{0}} e^{-4 l \gamma}+\ldots\right.
\end{aligned}
$$

Since

$$
\rho_{i}=\frac{Z_{i}-z_{0}}{Z_{i}+Z_{0}}
$$

the voltage divider may be written as

$$
\frac{z_{i}}{z_{i}+z_{0}}=\frac{1+\rho_{i}}{2}
$$


Putting $\left(1+\rho_{S}\right) / 2$ into $(A-14)$ for the factor $Z_{S} /\left(Z_{S}+Z_{0}\right)$ obtains the result

$E_{s}(s)=E_{g} \frac{Z_{0}}{Z_{s}+Z_{0}}\left\{1+\rho_{r}\left(1+\rho_{s}\right) e^{-2 l \gamma}+\rho_{r}^{2} \rho_{s}\left(1+\rho_{s}\right) e^{-4 l \gamma}+\ldots\right.$

which is identical to that given by expansion of $(A-12)$. Therefore, it has been demonstrated that the RT diagram allows one to write down the equation for $E_{S}(s)$ directly by tracing out the signal flow while invoking the rules $1-4$.

Similarly, the received voltage $E_{r}(s)$ is obtained from the RT diagram as

$$
\begin{aligned}
& E_{r}(s)=\left[E_{g} \frac{Z_{0}}{Z_{s}+Z_{0}}\right]\left[e^{-l y} \frac{2 Z_{r}}{Z_{r}+Z_{0}}\right] \\
& \text { Gen to } A \text { AB } \\
& +\left[E_{g} \frac{Z_{o}}{Z_{s}+Z_{o}} e^{-\ell \gamma} \rho_{r} e^{-\ell \gamma} \rho_{s} e^{-\ell \gamma} \frac{2 Z_{r}}{Z_{r}+Z_{o}}\right]+\ldots \\
& =2 E_{g} \frac{Z_{0}}{Z_{s}+Z_{0}} \frac{Z_{r}}{Z_{r}+Z_{0}}\left[e^{-l \gamma}+\rho_{r} \rho_{s} e^{-3 l \gamma}+\ldots\right.
\end{aligned}
$$

which is identical to that given by expanding (A-13).

The RT diagram is a powerful tool for analyzing a discretely loaded uniform transmission line. Consider Fig. A-3 in which a uniform transmission line, characterized by $\mathrm{Z}_{0}(\mathrm{~s})$ and $\gamma(\mathrm{s})$, is 

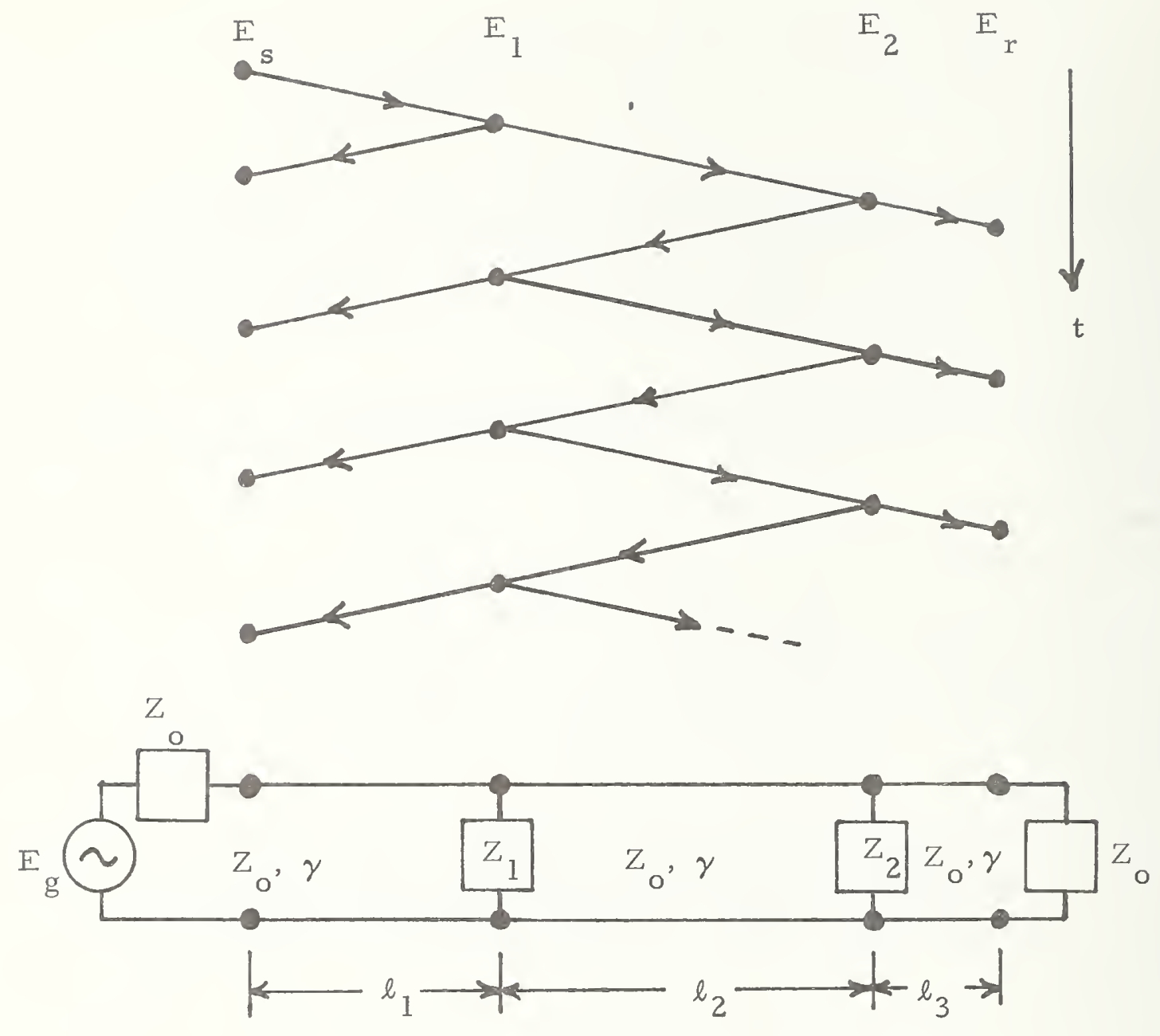

Figure A-3 The circuit and RT diagrams for a uniform transmission line terminated at each end in its characteristic impedance $Z_{0}(s)$ and discretely loaded by the impedances $Z_{I}(s)$ and $Z_{2}(s)$. 
discretely loaded by the shunt impedances $Z_{1}(s)$ and $Z_{2}(s)$ and doubly terminated in the characteristic impedance $Z_{0}(s)$. Consequently, there will be no reflections from the sending and receiving end terminations while internal reflections will exist due to the loading by $Z_{1}(s)$ and $\mathrm{Z}_{2}(\mathrm{~s})$.

Now it will be instructive to write out the initial voltage transmitted to node 2, $\left[E_{2}(s)\right]$, using the $R-T$ diagram rules. However, this time admittances will be used as their use is convenient for shunt loading. The change-over to admittances only requires $1 / Y$ substitution, e. g. , rule 1 becomes

$$
\begin{aligned}
\frac{Z_{0}(s)}{Z_{s}(s)+Z_{0}(s)} & =\frac{\left[Y_{0}(s)\right]^{-1}}{\left[Y_{0}(s)\right]^{-1}+\left[Y_{0}(s)\right]^{-1}} \\
& =\frac{Y_{s}(s)}{Y_{s}(s)+Y_{0}(s)}
\end{aligned}
$$

Similarly, rules 3 and 4 transform to the factors $2 Y_{0}(s)\left[Y_{0}(s)+Y_{r}(s)\right]^{-1}$ and $\left[Y_{0}(s)-Y_{r}(s)\right]\left[Y_{0}(s)+Y_{r}(s)\right]^{-1}$, respectively. Accordingly, tracing the initial path from node $g$ to node 2 , the initial voltage at node 2 is given by

$$
\begin{aligned}
\left.E_{\text {initial }}(s)\right]= & {\left[E_{g}(s) \frac{Y_{0}(s)}{Y_{0}(s)+Y_{0}(s)}\right]\left[\frac{2 Y_{0}(s)}{Y_{1}(s)+Y_{0}(s)} e^{-l_{1} \gamma(s)}\right] } \\
& {\left[\frac{2 Y_{0}(s)}{Y_{2}^{\prime}(s)+Y_{0}(s)} e^{-l_{2} \gamma(s)}\right] }
\end{aligned}
$$


where

$$
Y_{i}^{\prime}(s)=Y_{i}(s)+Y_{0}(s)
$$

because the total admittance at node $i$ seen by the advancing voltage wave is the sum of the discrete admittance $Y_{i}(s)$ and the characteristic admittance $Y_{0}(s)$ of the following transmission line segment. (A-14) reduces to

$$
\left[E_{2}(s)\right]_{\text {initial }}=\frac{E_{g}(s)}{2} \frac{2 Y_{0}(s)}{Y_{1}(s)+2 Y_{0}(s)} \frac{2 Y_{0}(s)}{Y_{2}(s)+2 Y_{0}(s)} e^{-\left(l_{1}+l_{2}\right) \gamma(s)}
$$

The initial voltage (A-15) exists for the time interval $0 \leq t \leq 2 \mathrm{~T}$ where

$$
T=\lim _{|s| \rightarrow \infty} \frac{l_{2} \gamma(s)}{s}=l_{2} \sqrt{L C}
$$

where $L$ and $C$ are the external inductance/m and capacitance/m of the uniform transmission line. As mentioned earlier $\gamma(\mathrm{s})$ can be written in the form $s T^{\prime}+\gamma^{\prime}(\mathrm{s})$; consequently, in the light of $(A-16)$ the limit of $\gamma^{\prime}(\mathrm{s})$ must vanish as $|\mathrm{s}| \rightarrow \infty$. This is the case as $\gamma^{\prime}(\mathrm{s})$ is the minimum phase part of $\gamma(s)$. 
FORM NBS-114A $(1.71)$

\begin{tabular}{|c|c|c|}
\hline $\begin{array}{c}\text { U.S. DEPT. OF COMM. } \\
\text { BIBLIOGRAPHIC DATA } \\
\text { SHEET }\end{array}$ & $\begin{array}{c}\text { 1. PUBLICATION OR REPORT NO. } \\
\text { NBSIR 73-330 }\end{array}$ & $\begin{array}{l}\text { 2. Gov't Accession } \\
\text { No. }\end{array}$ \\
\hline
\end{tabular}

4. TIT LE AND SUBTITLE

Frequency Domain Measurement of Baseband Instrumentation

7. AUTHOR(S) N. S. Nahman and R. M. Jickling

9. PERFORMING ORGANIZATION NAME AND ADDRESS

NATIONAL BUREAU OF STANDARDS, Boulder Labs. DEPARTMENT OF COMMERCE

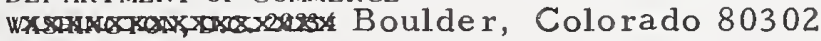

12. Sponsoring Organization Name and Address

Dept. of Defense, Calibration Coordination Group c/o Mr. M. L. Fruechtenicht AMSMI-M, Bldg. 5435

U.S. Army Metrology \& Calibration Center

Redstone Arsena1, Alabama 35809

15. SUPPLEMENTARY NOTES

16. ABSTRACT (A 200-word or less factual summary of most significant information. If document includes a significant bibliography or literature surve $y$, mention it here.)

Microwave measurement techniques were developed for characterizing the wideband feed-through sampling heads associated with time domain sampling oscilloscopes and frequency domain network analyzers. Such characterization or modeling is necessary for the removal of the oscilloscope distortion from the observed waveform to yield the input waveform; also, it is useful for extrapolation in estimating oscilloscope performance at higher frequencies.

The techniques were developed through measurements on a sampling oscilloscope having a 28 picosecond transition time (10\% to $90 \%$ ) and a $12.4 \mathrm{GHz}$ baseband bandwidth. The major results of the work are the development of voltage and impedance measurement techniques which provide the means for determining the sampling-head equivalent circuit parameters. The techniques are based upon slotted-1ine measurements and are not inherently limited to any particular frequency range. Experimental results were obtained for the sampling-head input impedance over the 7-12 GHz frequency range, and for the $10 \mathrm{GHz}$ sampling $100 \mathrm{p}$ impedance (vs. sampling-diode bias current).

17. KEY WORDS (Alphabetical order, separated by semicolons)

Bandwidth; diode; impedance; sampling; slotted line.

18. AVAILABILITY STATEMENT

$\mathrm{X}$ UNLIMITED.

FOR OFFICIAL DISTRIBUTION. DO NOT RELEASE TO NTIS.

$\begin{aligned} & \text { 19. SECURITY CLASS } \\
& \text { (THIS REPORT) }\end{aligned}$
\begin{tabular}{|l|l|} 
UNCL ASSIFIED & 21. NO. OF PAGES \\
\begin{tabular}{|l|l|} 
20. SECURITY CLASS \\
(THIS PAGE)
\end{tabular} & 22. Price \\
UNCL ASSIFIED & USCOMM-DC $66244-P 71$ \\
\hline
\end{tabular}




